\title{
Design Thinking, Digitalisierung und Diversity Management: Ein Praxisleitfaden für die Lehre
}

\author{
Katharina Gläsener \\ Thomas Afflerbach \\ Antje Ducki
}

Schriftenreihe „Gender-Dikurs“"

des Gender- und Technik-Zentrum (GuTZ)

der Beuth Hochschule für Technik Berlin

Band 10

Herausgeberinnen

Eva-Maria Dombrowski, Antje Ducki 

Design Thinking, Digitalisierung und Diversity Management: Ein Praxisleitfaden für die Lehre

Katharina Gläsener

Thomas Afflerbach

Antje Ducki

Schriftenreihe

des Gender- und Technikzentrum (GuTZ)

der Beuth Hochschule für Technik Berlin

Band 10

Herausgeberinnen

Eva-Maria Dombrowski, Antje Ducki 
(C) 2019 Dieses Werk ist im Verlag Barbara Budrich erschienen und steht unter folgender Creative Commons Lizenz: http://creativecommons.org/licenses/by-nc-nd/3.0/de/

Verbreitung, Speicherung und Vervielfältigung erlaubt, kommerzielle Nutzung und Veränderung nur mit Genehmigung des Verlags Barbara Budrich.

\section{(c) BY-NC-ND}

Dieses Werk steht im OpenAccess Bereich der Verlagsseite zum kostenlosen Download bereit (https://doi.org/10.3224/86388777).

Eine kostenpflichtige Druckversion (Printing on Demand) kann über den Verlag bezogen werden. Die Seitenzahlen in der Druck- und Onlineversion sind identisch.

ISBN 978-3-86388-777-3 (Druckfassung)

eISBN 978-3-86388-364-5 (eBook)

DOI $\quad 10.3224 / 86388777$

Druck: www.wirmachendruck.de

Verlag Budrich UniPress Ltd.

http://www.budrich-verlag.de 
Prof. Dr. Katharina Gläsener ist Gastprofessorin für das Thema Digitalisierung der Wirtschaft in einer multikulturellen Gesellschaft am Fachbereich Wirtschafts- und Gesellschaftswissenschaften der Beuth Hochschule für Technik Berlin. Im Mittelpunkt ihrer Forschung und Lehre stehen die Themen Digitalisierung, Diversity Management (insbesondere Sprache und Kultur), Personalmanagement, Teamarbeit, Innovationsmanagement, Empirische Forschungsmethoden sowie Macht und Mikropolitik. Zusätzlich bietet sie regelmäßig Workshops zu Design Thinking im hochschulischen und außerhochschulischen Bereich an.

Prof. Dr. Thomas Afflerbach ist Gastprofessor für Allgemeine Betriebswirtschaftslehre, insbesondere Dienstleistungsmanagement, an der Hochschule für Wirtschaft und Recht Berlin. Zusätzlich führt er als selbstständiger Innovationsberater regelmäßig Workshops durch, z. B. als Design Thinking Coach an der Hasso-Plattner-Institut Academy der Universität Potsdam. Seine Lehrund Forschungsschwerpunkte sind nutzer_innenzentriertes Innovationsmanagement (Design Thinking und Service Design), Marketing und Digitalisierung von Dienstleistungsunternehmen sowie moderne Arbeitswelten (z. B. virtuelle Teams und Vertrauen innerhalb von Organisationen), wobei er sich auf qualitative Forschungsmethoden spezialisiert hat.

Prof. Dr. Antje Ducki ist Professorin für Arbeits- und Organisationspsychologie an der Beuth Hochschule für Technik Berlin. Zusammen mit Prof. Dr. Eva Maria Dombrowski leitet sie seit 2009 das Gender- und Technik-Zentrum (GuTZ). Im Mittelpunkt ihrer Forschungsarbeiten stehen die Themen betriebliche Gesundheitsförderung, Mobilität und Gesundheit sowie verschiedene Genderund Diversity-Themen.

Gefördert durch das

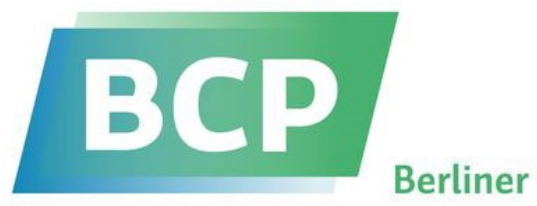

\section{Chancengleichheitsprogramm}

Diese Publikation basiert auf einer Analyse- und Recherchetätigkeit, die über das Berliner Chancengleichheitsprogramm für Frauen in Forschung und Lehre gefördert wurde.

Impressum

Herausgeberinnen:

Prof. Dr. Eva-Maria Dombrowski

Prof. Dr. Antje Ducki

Redaktion:

Gender- und Technik-Zentrum der

Beuth Hochschule für Technik

Luxemburger Str. 10

13353 Berlin

E-Mail: gutz@beuth-hochschule.de

Internet: https://www.beuth-hochschule.de/gutz/

Verantwortlich für den Inhalt sind die Autor_innen. 



\section{Inhaltsverzeichnis}

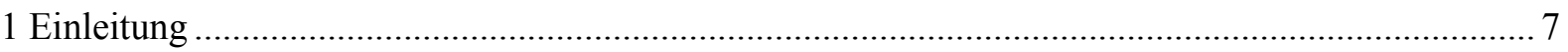

2 Theoretische Grundlagen zu Design Thinking, Digitalisierung und Diversity Management .............. 7

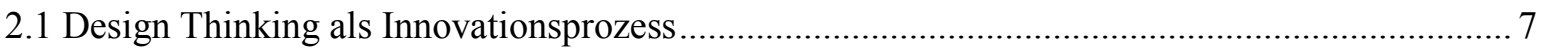

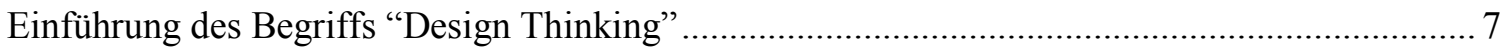

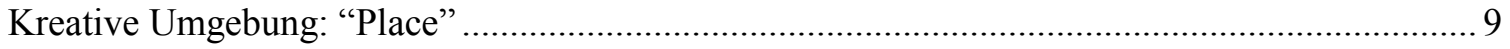

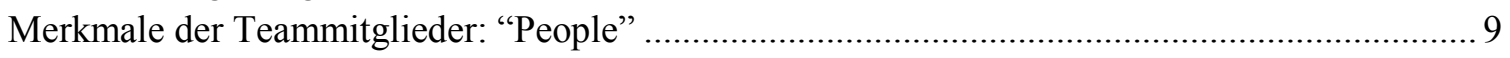

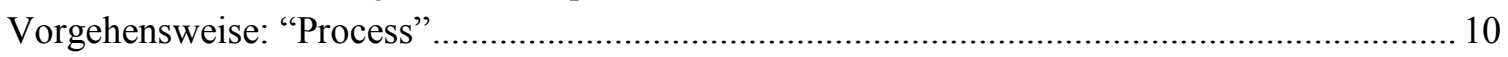

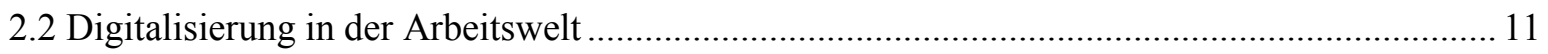

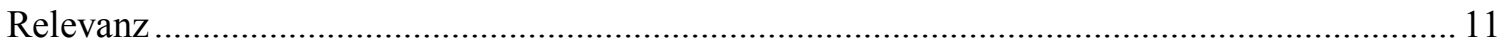

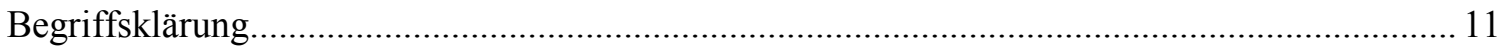

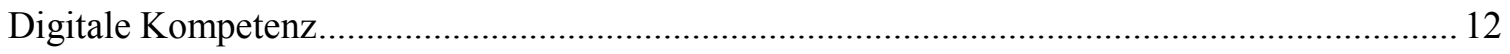

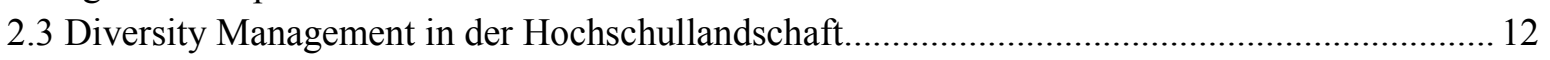

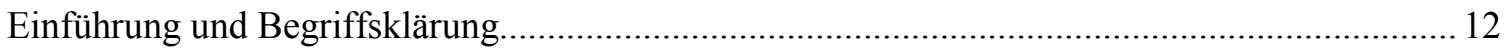

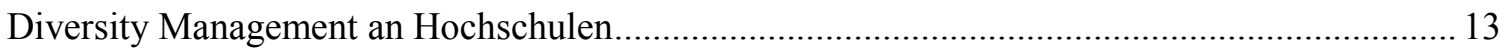

3 Das Fallbeispiel: Design Thinking, Digitalisierung und Diversity Management in der

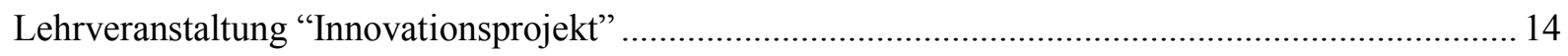

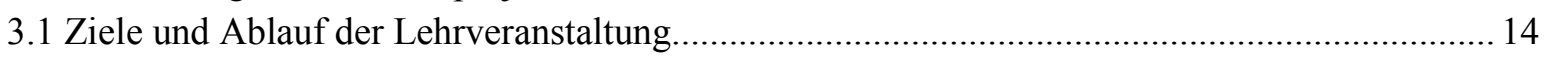

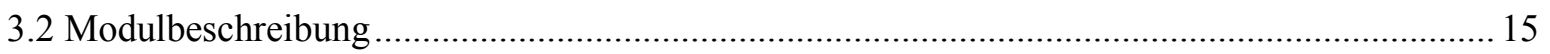

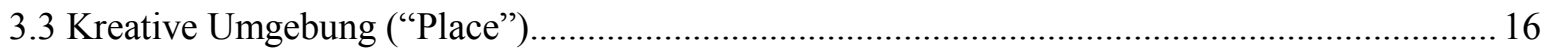

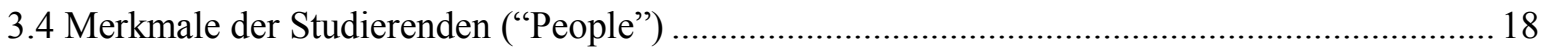

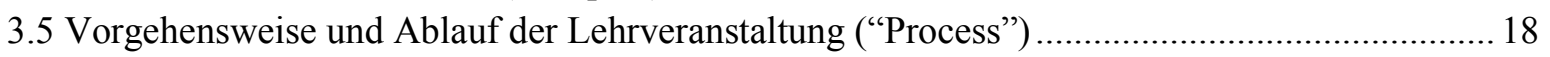

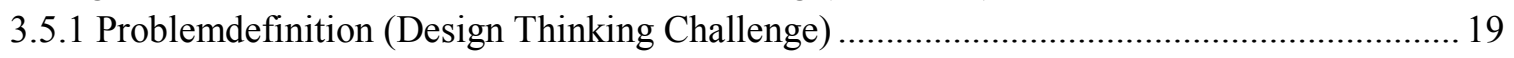

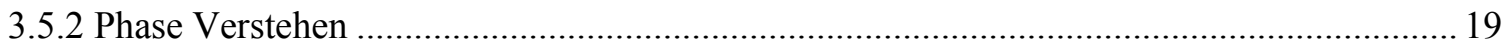

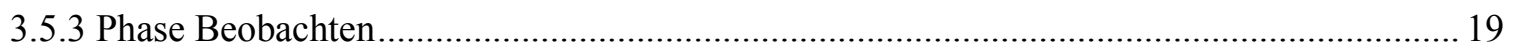

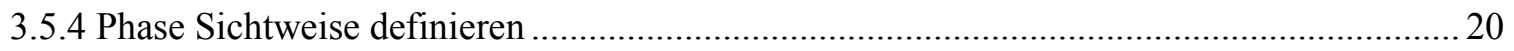

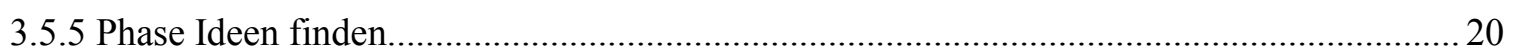

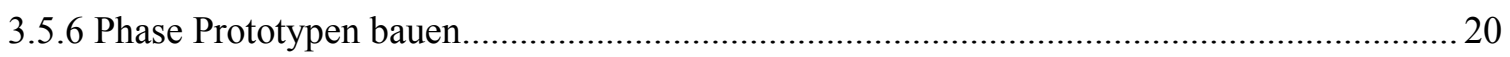

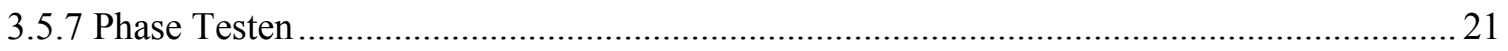

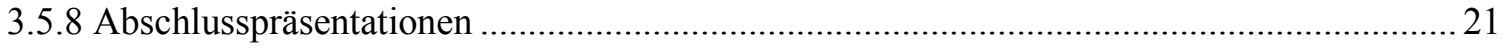

4 Ergebnisse: Innovative Ideen zur digitalen Unterstützung von Vielfalt an der Beuth Hochschule für

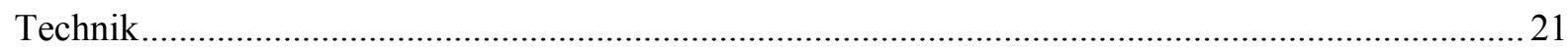

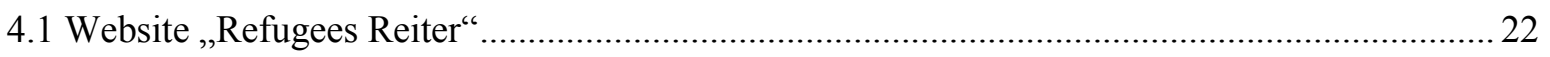

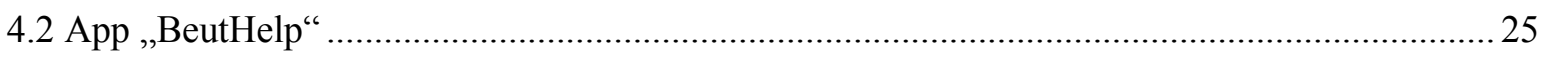

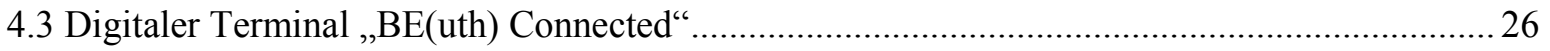

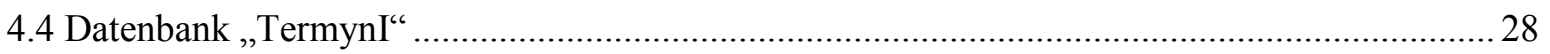

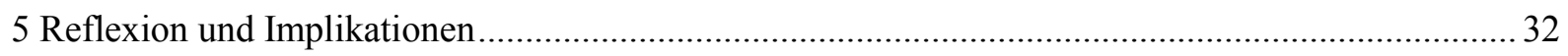

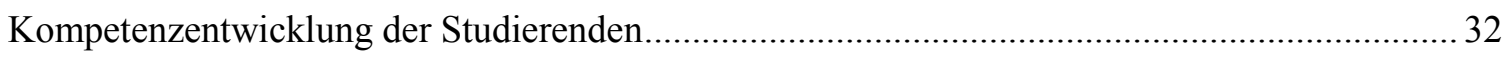

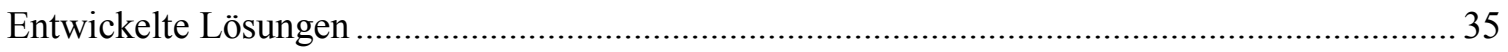

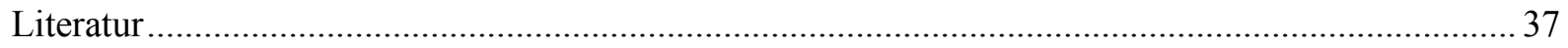

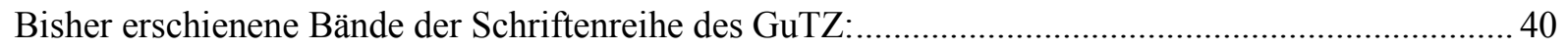

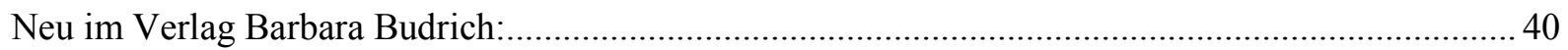





\section{Einleitung}

Design Thinking, Digitalisierung und Diversity Management sind zentrale Bestandteile der heutigen Arbeitswelt. So sollten auch alle drei Themen an der Hochschule an Bedeutung gewinnen und Bestandteil des Studiums sein. In der Lehrveranstaltung "Innovationsprojekt: Digitalisierung und Diversity Management", die zum ersten Mal im Sommersemester 2017 an der Beuth Hochschule für Technik Berlin angeboten wurde, werden diese drei Themen miteinander kombiniert und sind Bestandteil der fachlichen, methodischen, persönlichen und sozialen Kompetenzbildung der Studierenden. Diese Ausbildung ermöglicht es den Studierenden in einem Semester im Rahmen eines Studium Generale-Kurses grundlegende Kenntnisse in den Themen Design Thinking, Digitalisierung und Diversity Management zu erwerben. Auf Basis der einsemestrigen Erfahrung wird in diesem Beitrag beschrieben:

(1) wie die Studierenden ihre Kompetenzen in den Themen Design Thinking, Digitalisierung und Diversity Management weiterentwickeln konnten;

(2) welche Lösungen die Studierenden zu dem Thema digitale Unterstützung von Vielfalt an der Beuth Hochschule für Technik Berlin erarbeitet haben.

Der vorliegende Beitrag versteht sich als Beitrag zu einer Diskussion, die auch im hochschulischen Kontext geführt werden muss: Wie wollen und werden wir zukünftig arbeiten? Der Text gliedert sich in fünf Kapitel. Nach dieser Einleitung werden in Kapitel 2 die theoretischen Grundlagen zu den drei Themenkomplexen Design Thinking, Digitalisierung und Diversity Management gelegt. Kapitel 3 dient dazu, das verwendete Fallbeispiel - die Lehrveranstaltung "Innovationsprojekt: Digitalisierung und Diversity Management" an der Beuth Hochschule für Technik Berlin - zu erläutern und gleichzeitig Umsetzungshinweise für andere Lehrende und Interessierte in der Erwachsenenbildung zu geben. In Kapitel 4 werden die innovativen Ideen der Studierenden zur digitalen Unterstützung von Vielfalt an der Beuth Hochschule für Technik Berlin vorgestellt, bevor in Kapitel 5 diese Lösungen von den Autor_innen reflektiert und Implikationen für die Themen Design Thinking, Digitalisierung und Diversity Management an der Hochschule diskutiert werden.

\section{Theoretische Grundlagen zu Design Thinking, Digitalisierung und Diversity Management}

Die Begriffe Design Thinking, Digitalisierung und Diversity Management umfassen in Bezug auf die Gestaltung von Lehrveranstaltungen verschiedene Aspekte, die in den nachfolgenden Abschnitten erläutert werden.

\subsection{Design Thinking als Innovationsprozess}

Design Thinking ist ein Terminus, welcher noch vor einigen Jahren in Deutschland kaum bekannt war, der aber mittlerweile als eine Art 'Panazee' (mythisches Universal-Heilmittel) in vielen Unternehmen, Organisationen, aber auch Bildungseinrichtungen, Einzug gehalten hat (Meinel, Weinberg \& Krohn 2015, S. 11).

\section{Einführung des Begriffs "Design Thinking"}

Design Thinking ist eine agile Innovationsmethode, welche weltweit an Popularität gewinnt. Es gibt keine einheitliche Definition, aber eine Vielzahl an wissenschafts- oder praxisorientierten Begriffsbestimmungen von Design Thinking, die unterschiedliche Aspekte und Sichtweisen hervorheben. Exemplarisch werden nachfolgend zwei Definitionen vorgestellt: 
- "Design Thinking [...] ist erfinderisches Denken mit radikaler Kunden- beziehungsweise Nutzerorientierung. Es basiert auf dem Prinzip der Interdisziplinarität und verbindet in einem strukturierten, moderierten Iterationsprozess die Haltung der Ergebnisoffenheit mit der Notwendigkeit der Ergebnisorientierung. [...] Dabei vereint der Ansatz viele Elemente [...] zu einer neuen, sich stets weiterentwickelnden Innovationsmethode" (Erbeldinger \& Ramge 2015, S. 13).

- "Design Thinking ist eine Innovationsmethode, die auf Basis eines iterativen Prozesses nutzer- und kundenorientierte Ergebnisse zur Lösung von komplexen Problemen liefert" (Uebernickel et al. 2015, S. 16).

Es lässt sich festhalten, dass es bei Design Thinking um ein systematisches Innovieren mit Fokus auf Nutzer_innenbedürfnisse geht. Neuerungen sollen strukturiert erstellt und Lösungen für (komplexe) Probleme entwickelt werden, wobei das Ergebnis zunächst offen beziehungsweise nicht vorab spezifiziert ist. Damit kann Design Thinking sowohl als Denkweise ("Mindset"), Prozess oder Werkzeugkoffer ("Toolbox") verstanden werden (Brenner, Uebernickel \& Abrell 2016, S. 3). Durch das iterative Vorgehen, welches an dem_der Nutzer_in und dessen_deren Bedürfnissen orientiert ist, wird Design Thinking den agilen Methoden zugeordnet (Link 2014, S. 65f.).

Seinen Ursprung hat Design Thinking als Denk- und Arbeitsansatz, laut Stanford-Professor David Kelley, dem Gründer der "d.school", in der Bauhausbewegung in Deutschland. Durch die Gründung eines Bauhauses in den 1920er Jahren hatte der Architekt Walter Gropius einen ersten Versuch unternommen, verschiedene fachliche Disziplinen, wie Architektur, Kunst, Theater, Musik und Gestaltung, zusammenzuführen, um damit die Lösungskompetenz für komplexe Fragestellungen zu steigern und eine größere Vielfalt an Möglichkeiten zu eröffnen (Meinel, Weinberg \& Krohn 2015, S. 11f.).

Folglich sollte auch Design in Design Thinking nicht zu begrenzt, als - salopp formuliert - "Dinge schöner machen" verstanden werden. Während im deutschsprachigen Raum der Begriff Design meist mit einer „formgerechten und funktionalen Gestaltgebung und daraus sich ergebende Form eines Gebrauchsgegenstandes o.Ä.“ (Duden o.J.) assoziiert wird, wird der Begriff Design im englischen Sprachraum vielschichtiger verstanden. Beim englischsprachigen Begriffsverständnis fließt zusätzlich die konzeptionelle und technische Gestaltung von Objekten und Systemen mit ein und vergrößert den Umfang der Begriffsbedeutung (Uebernickel et al. 2015, S. 16). Der Begriff ist hierbei sogar umfänglicher zu verstehen als Design im Sinne der strukturierten Suche nach kreativen Lösungen für komplexe Probleme (Gürtler \& Meyer 2016, S. 6). Denn der Denk- und Arbeitsansatz von Design Thinking nimmt explizit Bezug auf die Arbeitsweise von Designer_innen. Diese entwickeln Ideen und suchen konkrete und umsetzbare Lösungen (Produkte, Dienstleistungen, Systeme) für komplexe Probleme, welche so nützlich wie möglich für eine spezielle Nutzer_innengruppe sind.

Um sich derartigen komplexen Problemen zu widmen, haben sich im Design im Allgemeinen und Design Thinking im Speziellen charakteristische Arbeits- und Lernstrategien entwickelt: kognitive Muster, um sowohl verschiedenartiges Wissen und verschiedenartige Perspektiven von Anderen zu erfassen als auch dieses Wissen in neue Dienstleistungs- und/oder Produkterlebnisse umzuwandeln und zu synthetisieren. Aufgrund dieser kognitiven Strategien spricht man auch von Design Thinking und von der Ausgestaltung von Erlebnissen als bewussten Prozess (Brown 2009; Dunne \& Martin 2006; Lindberg, Meinel \& Wagner 2011). 
Kernelemente von Design Thinking: Die drei Ps aus Place, People und Process

Aus dem oben genannten Verständnis von Design Thinking ergeben sich folgende Kernelemente: (1) Kreative Umgebung ("Place"), (2) Merkmale der Teammitglieder ("People") und (3) Vorgehensweise ("Process").

Kreative Umgebung: "Place"

Design Thinking sollte in einer kreativen Umgebung stattfinden, denn der bewusste Umgang mit Räumlichkeiten sowie deren Nutzung für die Teamarbeit bilden ein Grundelement. Eine kreative Umgebung zeichnet sich durch eine inspirierende und ideenförderliche Einrichtung und Gestaltung aus (Schallmo 2017, S. 20). Da jeder Arbeitsmodus unterschiedliche Anforderungen an das Team stellt, sollte die Umgebung dementsprechend flexibel sein, um diesen Anforderungen gerecht zu werden (Gürtler \& Meyer 2016, S. 20f.). Diese ist gekennzeichnet durch eine offene und flexibel anpassbare Raumgestaltung. Unterstützend dafür sind Möbel, die den kollaborativen Prozess der Teamarbeit unterstützen, idealerweise verschiebbare Möbel auf Rollen (Blatt \& Sauvonnet 2017, S. 25).

In Abbildung 1 ist beispielhaft das Arbeitsumfeld an der School of Design Thinking des HassoPlattner-Instituts in Potsdam aufgezeigt.

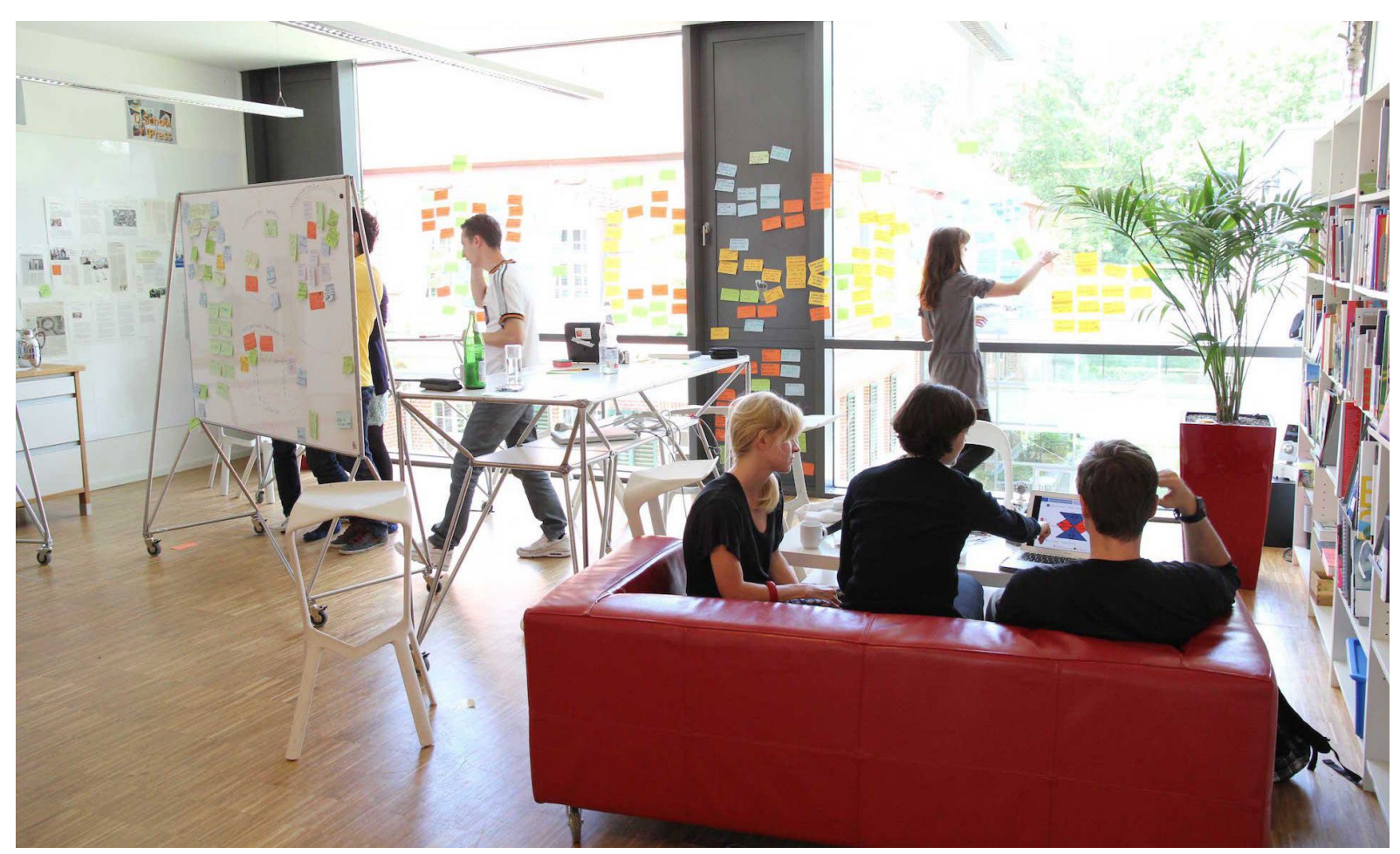

Abbildung 1: Arbeitsumfeld an der School of Design Thinking

(Quelle: Hasso-Plattner-Institut School of Design Thinking 2018)

Merkmale der Teammitglieder: "People"

Ein weiteres zentrales Element von Design Thinking ist der multidisziplinäre, teambasierte Ansatz, denn "Lösungen entstehen heute und in Zukunft im Team, sie basieren auf interdisziplinären oder multidisziplinären Denken und Handeln" (Weinberg 2015, S. 161). Design Thinking setzt auf eine kollaborative Innovationskultur, bei der die Teammitglieder ihre verschiedenen Perspektiven und Expertisen in den Problemlösungsprozess einbringen können. Es ist dabei förderlich, wenn die einzelnen Teammitglieder selbst Fähigkeiten und Erfahrungen in mehr als einer Disziplin besitzen (Brown 2009, S. 3). Hintergrund dafür ist die an der School of Design Thinking der Universität 
Stanford entwickelte Idee von Innovationskultur, die davon ausgeht, dass die innovativsten Lösungen in multidisziplinären Teams entstehen.

Gemäß dem Motto "all of us are smarter than any of us" (Brown 2009, S. 26) arbeiten im Design Thinking mehrere Menschen in Teams gemeinschaftlich an einer Aufgabe. Dabei können sich die einzelnen Teammitglieder aufgrund ihrer unterschiedlichen Perspektiven, Sichtweisen und Erfahrungswerte gegenseitig bereichern und fördern, aber gleichzeitig auch herausfordern und inspirieren (Gürtler \& Meyer 2016, S. 19). Das Team kann dabei beispielsweise aus verschiedenen Disziplinen oder Hierarchieebenen zusammengesetzt werden (Grots \& Pratschke 2009, S. 19). Dabei sind nicht nur die fachlichen Spezialisierungen der einzelnen Teammitglieder maßgebend, sondern auch soziale Dimensionen wie beispielsweise Geschlecht, Altersgruppen und soziale Hintergründe. Design Thinking Teams sind idealerweise möglichst durchmischt und vielfältig konstituiert, um differenzierte Blickwinkel auf die Problemstellung zu gewährleisten (Blatt \& Sauvonnet 2017, S. 24). Gleichzeitig stellt diese Vielfalt in der Teamzusammensetzung auch eine besondere Herausforderung dar. Es benötigt Menschen, welche gefestigt in ihrem Fachwissen sind, und bereit sind, sich auf andere Bereiche einzulassen und interdisziplinär zu arbeiten.

Vorgehensweise: "Process"

Ein Design Thinking Projekt startet mit der Definition der Design Challenge und der intensiven Auseinandersetzung mit dieser Herausforderung als Team. In dieser Phase des Problembereichs (Prozessschritt 1: Verstehen) geht es darum, als Team möglichst viel über das eigentliche Problem zu lernen und es möglichst gut und ganzheitlich zu verstehen. Dafür bieten sich im nächsten Schritt (Prozessschritt 2: Beobachten) diverse - aus der empirischen Sozialforschung bekannte - Methoden an, wie Interviews mit potenziellen Nutzer_innen bzw. Betroffenen führen. In der darauf folgenden Phase (Prozessschritt 3: Sichtweise definieren) geht es darum, aus der Vielzahl der gesammelten Informationen, diejenigen zu extrahieren, auf die sich das Design Thinking Team in den nächsten Schritten des Prozesses konzentrieren möchte. Dabei wird sich auf eine_n konkrete_n Nutzer_in mit den jeweiligen Bedürfnissen fokussiert, um als Sprungbrett für den Wechsel in den Lösungsbereich zu fungieren. In der ersten Phase des Lösungsbereichs (Prozessschritt 4: Ideen finden) wird nach einer Quantität an Ideen gestrebt. Einige dieser Ideen werden anschließend in Prototypen konkretisiert (Prozessschritt 5: Prototypen entwickeln). Diese prototypisch umgesetzten Lösungen werden dann wiederum zügig mit potentiellen Nutzern und Nutzerinnen getestet (Prozessschritt 6: Testen), um somit nicht nur mehr Empathie für die Zielgruppe zu entwickeln, sondern auch, um mit deren Testfeedback die Lösung weiterzuentwickeln. Eine derartige kontinuierliche Rückkopplung mit potenziellen Nutzer_innen mittels des wiederholten Testens der Lösungsideen anhand von Prototypen, ermöglicht die benötigten Lernzyklen, um schnell und ressourcenschonend dem Ziel von nutzer_innenzentrierten Lösungen näher zu kommen.

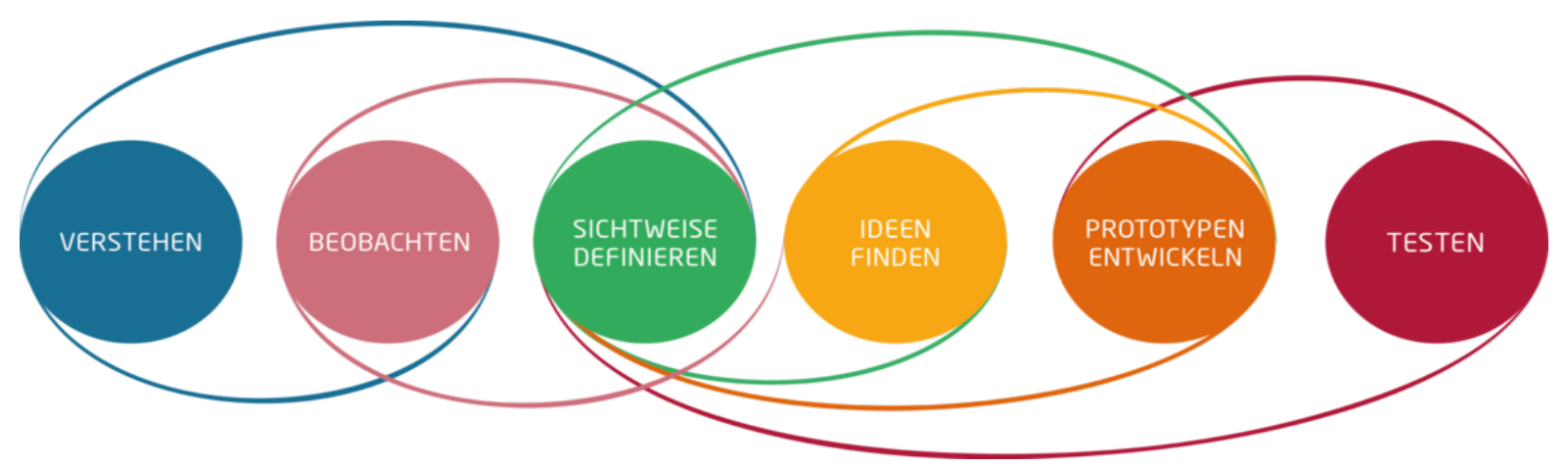

Abbildung 2: Design Thinking Prozess

(Quelle: Hasso-Plattner-Institut Academy 2018) 
Obwohl der Design Thinking Prozess mit dieser Beschreibung vermeintlich sequenziell wirkt, ist er tatsächlich iterativ (siehe Abbildung 2). In einem Design Thinking Prozess findet ein häufiger Wechsel zwischen Phasen des Problemverstehens, der Ideenfindung und der Lösungsvalidierung statt. Mit jeder Iterationsschleife kann und soll die Lösung verfeinert werden (Gürtler \& Meyer 2016, S. $31)$.

\subsection{Digitalisierung in der Arbeitswelt}

Die Digitalisierung verändert sowohl unser Sozial- als auch Arbeitsleben grundlegend, weshalb ein kompetenter Umgang mit digitalen Technologien für alle Menschen entscheidend für eine erfolgreiche Teilhabe ist.

\section{Relevanz}

Die Digitalisierung hat einen nachhaltigen Einfluss auf Wirtschaft und Gesellschaft (Weigert, Bruhn \& Strenge 2017, S. 325). Weil die Digitalisierung alle Aspekte unseres privaten und professionellen Lebens betrifft, haben sowohl Führungskräfte als auch politische Entscheidungsträger_innen das Thema weit oben auf ihrer Prioritätenliste gesetzt. Die Vor- und Nachteile des Digitalisierungsprozesses werden ausführlich in den Medien und auf Konferenzen diskutiert (Legner et al. 2017, S. 301). Dabei greift die digitale Transformation tief in das Fundament des unternehmerischen Handelns ein und führt $\mathrm{zu}$ einem tiefgreifenden Wandel der Unternehmensstrategie, -struktur, -prozesse und auch -kultur (Ducki 2016). Die Digitalisierung beschreibt damit einen dauerhaften - in Teilen sogar disruptiven - Prozess und verändert die Arbeitswelt aller, mit weitreichenden Konsequenzen für den globalen Arbeitsmarkt (Weigert, Bruhn \& Strenge 2017, S. 325 f.). Mit der zunehmenden Digitalisierung am Arbeitsplatz sind moderne Informations- und Kommunikationsmittel in unserem Arbeitsleben unverzichtbar (Johns \& Gratton 2013). Der Laptop und das Smartphone gehören heutzutage zur Standardausstattung nahezu aller Berufe (Bitkom 2015, S. 7). Auch die private Nutzung von Informations- und Kommunikationstechnologien erreicht mittlerweile in Deutschland eine erhebliche Durchdringung des Alltags. Zwischen 2005 und 2015 ist der Anteil der Nutzer_innen eines Computers oder Laptops von $70 \%$ auf $83 \%$ gestiegen (BPB 2016).

\section{Begriffsklärung}

Interessanterweise gibt es im englischen Sprachgebrauch eine begriffliche Differenzierung zwischen "Digitization" und "Digitalization" - eine Differenzierung, welche es in dieser Form in der deutschen Sprache nicht gibt und einheitlich mit "Digitalisierung" übersetzt wird. "Digitization" beschreibt den technischen Prozess der Umwandlung von analogen Signalen in ihre digitale Form, welche im Endeffekt aus Binärziffern bestehen (Tilson et al. 2010, S. 748 ff.; Legner et al. 2017, S. 301). Hierzu sagen sowohl Internetpionier_innen als auch Digitalisierungsexperten_innen, „dass alles, was man digitalisieren kann, auch digitalisiert werden wird“; es sei eben nur eine Frage der Zeit, wie Bundeskanzlerin Angela Merkel bereits im Jahr 2013 bemerkte (Weigert, Bruhn \& Strenge 2017, S. 327). "Digitalization" beschreibt hingegen die vielschichtigen soziotechnischen Phänomene und Prozesse des Übernehmens und Nutzen von Technologien im umfassenderen individuellen, organisationalen oder gesellschaftlichen Kontext (Legner et al. 2017, S. 301). Damit einher gehen veränderte Verhaltensweisen und neue Anforderungen an Arbeitnehmer_innen und Unternehmen. Der vermehrte Einsatz neuer Informations- und Kommunikationstechnologien ermöglicht und erfordert neue Formen der Organisation sowie Strukturierung von Arbeit (De Leede \& Kraijenbrink 2014; Afflerbach \& Gläsener 2016). Mit der zunehmenden Verbreitung von Smartphones können Mitarbeitende heutzutage zu jeder Zeit an (fast) jedem Ort arbeiten (Derks \& Bakker 2010). Die letztere Auslegung von "Digitalisierung" - also "Digitalization" - ist das Verständnis, welches wir in dem vorliegenden Beitrag vertreten. 


\section{Digitale Kompetenz}

Wenn die Digitalisierung für alle Aspekte unseres privaten und professionellen Lebens relevant ist, dann wird Digitale Kompetenz zu einer wichtigen Schlüsselkompetenz - sowohl für das Individuum als auch für das Unternehmen. Allgemein beschreibt der Begriff Kompetenz die Fähigkeiten eines einzelnen Menschen, die sich durch die eigenständige Bewältigung von spezifischen Aufgaben innerhalb eines Handlungskontexts verdeutlichen. Auf die Arbeitswelt übertragen bedeutet Kompetenz demnach, dass Mitarbeiter_innen die generelle Voraussetzung besitzen, die berufsbezogenen Anforderungen situationsübergreifend $\mathrm{zu}$ bewältigen (Meifert 2013). Darauf aufbauend wird in der Begriffsdefinition der Deutschen Gesellschaft für Personalführung (DGFP) "Digitale Kompetenz” wie folgt erläutert: „Digitale Kompetenzen sind (neue) Fähigkeiten, die Mitarbeiterinnen und Mitarbeiter in die Lage versetzen, digitale Technologien anzuwenden, im Rahmen ihres Aufgabenprofils zu nutzen und darüber hinaus die digitale Transformation von Geschäftsprozessen mit voranzutreiben“"(DGFP 2016, S. 8).

Soziale und wirtschaftliche Teilhabe in der Zukunft ist ohne digitale Teilhabe kaum mehr denkbar. Diese Entwicklung erfordert neue Aus- und Weiterbildungskonzepte. Schule, Ausbildung und Hochschule müssen die Schüler_innen, Auszubildenden und Studierenden auf das digitalisierte Arbeitsleben vorbereiten, da eine digitale Teilhabe heutzutage eine Voraussetzung zur wirtschaftlichen und sozialen Integration ist (BPB 2016).

Digitale Kompetenz wird am einfachsten erlernt, indem Schüler_innen, Auszubildende, Studierende aber auch Arbeitnehmer_innen und Führungskräfte in Wirtschaft und Wissenschaft sich bewusst und kritisch mit den verschiedenen Aspekten der Digitalisierung auseinandersetzen. "Digitale Fitness" als Facette der oben genannten Digitalen Kompetenz beschreibt einen wichtigen Teilaspekt dieser Schlüsselkompetenz, die ein grundsätzliches Verständnis der technologischen Möglichkeiten und Einsatzmöglichkeiten beschreibt. Durch das Wissen, welche digitalen Geräte (Tools, Apps etc.) für welche Aufgaben am besten geeignet sind, um den Arbeitsvorgang bestmöglich zu unterstützen und zu erleichtern, entsteht eine fundierte Entscheidungsfähigkeit jedes einzelnen Individuums (DGFP 2016, S. 14). Zur Digitalen Kompetenz gehört darüber hinaus auch den eigenen Umgang mit digitalen Medien zu reflektieren und bewusst zu steuern.

\subsection{Diversity Management in der Hochschullandschaft}

Hochschulen sehen sich einer immer größer werdenden Diversität ihrer Angehörigen gegenübergestellt. Studierende, Mitarbeiter_innen und Lehrende kommen zunehmend aus dem Ausland, sprechen unterschiedliche Muttersprachen oder sind in anderer Art und Weise vielfältig. Seit 2015 gibt es in Deutschland auch immer mehr Studierende mit einem Fluchthintergrund. Die Zahl ausländischer Studierender in Deutschland (Bildungsausländer_innen) beläuft sich für das Studienjahr 2017 auf rund 13\% (Statista 2018). Der Anteil ausländischer Studierender in Berlin liegt 2017 sogar bei 15\% (DAAD 2018, S. 55). Einige Hochschulen reagieren auf diese sich ändernden Bedingungen mit einem strategisch geplanten Diversity Management, um ihrer gesellschaftlichen Verantwortung, Orte des diversitätsgerechten Lehrens und Lernen zu sein, gerecht zu werden (Auferkorte-Michaelis \& Linde 2016, S. 803).

\section{Einführung und Begriffsklärung}

Diskriminierungsfreies Zusammenarbeiten unterschiedlicher Menschen steht verstärkt auf der politischen Agenda. In Deutschland ist das Thema unter Begriffen wie Antidiskriminierung, Chancengerechtigkeit, Gleichstellung, Inklusion aber auch zunehmend unter dem Begriff Diversity Management bekannt. 
Der Begriff Diversity beschreibt die Verschiedenheit, Vielfalt und Mannigfaltigkeit von Menschen und bezieht sich dabei auf eine Bandbreite an Merkmalen und Charakteristika. Als relevante Diversity-Merkmale werden in der deutschsprachigen Literatur zumeist Geschlecht, Nationalität, Alter, Behinderung, Religion und sexuelle Orientierung thematisiert (Krell et al. 2007; Lederle 2007). Des Weiteren können weitere Merkmale wie beispielsweise Familienstand oder sozialer und sozioökonomischer Hintergrund hinzu zählen. Mit dem Begriff Diversity Management werden dementsprechend "Strategien, Programme und Maßnahmen für einen konstruktiven und produktiven Umgang mit Vielfalt" (Krell et al. 2007, S. 9) bezeichnet. Diversity Management ist somit ein strategisches Element der Organisationsleitung (Aretz \& Hansen 2003).

\section{Diversity Management an Hochschulen}

In Deutschland werden nicht nur Unternehmen, sondern auch Hochschulen zunehmend diverser. Die aktuelle Debatte um Diversity an deutschen Hochschulen speist sich insbesondere aus zwei Quellen: Erstens, Diversity Management verspricht an Hochschulen zahlreiche Vorteile als Imagefaktor und zur Gewinnung der "besten Köpfe" (Bender, Schmidbaur \& Wolde 2013, S. 7). Als Konzept, das u.a. impliziert, demographischen Entwicklungen und bevorstehendem Studierenden- und Fachkräftemangel entgegenzuwirken, verfolgt Diversity Management das Ziel, die geeigneten Studierenden und Mitarbeiter_innen auf internationaler Ebene, sowie aus nicht-traditionellen bzw. schwer erreichbaren Zielgruppen anzuwerben und zu rekrutieren, wie aktuell beispielsweise Geflüchtete. Dieser Verständnisansatz von Diversity entspricht der "access-and-legitimacy perspective" der US-amerikanischen Organisationsforscherin Robin J. Ely und ihres Kollegen David A. Thomas (Ely \& Thomas 2001, S. 243 ff.). Bei diesem Marktzutritts- und Legitimitätsansatz stehen ökonomische Argumente im Vordergrund - Diversität wird somit zum Wettbewerbsvorteil (Klein 2013). Zweitens, Diversity Management wird an Hochschulen im Kontext von moralischen Argumenten verfolgt (Bender, Schmidbaur \& Wolde 2013, S. 7). Das Ziel Bildungs- und Chancengerechtigkeit herzustellen ist demnach eine weitere Begründung für die Umsetzung eines Diversity Managements an deutschen Hochschulen (Auferkorte-Michaelis \& Linde 2016, S. 805). Beide Stränge vermengen sich in der Politik und bauen unter anderem auf der von der Hochschulrektorenkonferenz geforderten "Hochschule für alle" auf (Hochschulrektorenkonferenz 2009). Auslöser für ein Diversity Management ist demnach häufig der Veränderungsdruck durch die Politik. Der Wissenschaftsrat hat 2013 beispielsweise gefordert, dass Hochschulen strategisch auf die Heterogenität der Studierendenschaft reagieren müssen (Wissenschaftsrat 2013, S. 32 ff.). Der Veränderungsdruck in den Hochschulen wächst, insbesondere da seit der Jahrtausendwende nach der europäischen Hochschulreform Hochschulen zunehmend veranlasst werden, ihre Prinzipien und Leitlinien zu explizieren und sie sich immer wieder neuen politischen Vorgaben gegenüber gestellt sehen (Auferkorte-Michaelis \& Linde 2016, S. 807). Eine bildungspolitische Förderung begünstigt dabei die Umsetzung von Diversity Management in finanzieller und struktureller Hinsicht (ebenda, S. 805).

Die Ernsthaftigkeit, mit der eine Hochschule Diversity Management betreibt, wird in einer DiversityStrategie oder einem Diversity-Leitbild erkennbar. Diversity-Konzepte sind mittlerweile auch zum Gegenstand des Wettbewerbs der Hochschulen untereinander geworden (Klein 2013). Für eine umfassende Verankerung von Diversity Management in Hochschulen wird ein Gesamtkonzept benötigt, welches unter anderem Ansätze wie Gender Mainstreaming oder der interkulturellen Öffnung integriert (Auferkorte-Michaelis \& Linde 2016, S. 808). Mit der Entwicklung und Umsetzung einer Diversity-Strategie können Hochschulen der gesellschaftlichen Herausforderung begegnen und sich der gesellschaftlichen Verantwortung stellen, Orte eines diversitätsgerechten Lehrens und Lernen zu sein. 


\section{Das Fallbeispiel: Design Thinking, Digitalisierung und Diversity Management in der Lehrveranstaltung "Innovationsprojekt"}

In den nachfolgenden Abschnitten wird die Gestaltung und Umsetzung von Design Thinking, Digitalisierung und Diversity Management in der Lehrveranstaltung "Innovationsprojekt" erläutert, bevor in Kapitel 4 die von den Studierenden erarbeiteten Lösungen beschrieben werden.

\subsection{Ziele und Ablauf der Lehrveranstaltung}

Im Sommersemester 2017 wurde an der Beuth Hochschule für Technik Berlin zum ersten Mal die Lehrveranstaltung "Innovationsprojekt: Digitalisierung und Diversity Management" durchgeführt. Das Konzept für diese Lehrveranstaltung wurde von der Erstautorin, Gastprofessorin am Fachbereich Wirtschafts- und Gesellschaftswissenschaften der Beuth Hochschule für Technik Berlin, entwickelt und wird seitdem jedes Semester als allgemeinwissenschaftliche Ergänzung im Studium Generale angeboten. Beim Studium Generale handelt es sich um ein fachbereichsübergreifendes Wahlpflichtangebot für alle Bachelor- und Masterstudierenden an der Beuth Hochschule für Technik Berlin. Je nach Studiengang müssen die Studierenden im Laufe ihres Studiums zwei oder vier Lehrveranstaltungen im Studium Generale absolvieren.

Inhaltlich dienen die Lehrveranstaltungen im Studium Generale der Ausprägung der fachlichen, methodischen, persönlichen oder sozialen Kompetenz der Studierenden (Beuth Hochschule 2018a). Da Kompetenzen nicht nur knapp, sondern auch wertvoll, meist dauerhaft, häufig begrenzt imitierbar, schlecht übertragbar und beschränkt substituierbar sind, sind nicht nur Unternehmen bestrebt, gezielt Kompetenzen bei ihren Mitarbeitern und Mitarbeiterinnen aufzubauen (Kauffeld, Grote \& Frieling 2011), sondern auch die Studierenden an der Beuth Hochschule für Technik Berlin können sich durch den Erwerb von Kompetenzen als zukünftige Arbeitnehmer_innen profilieren. Bei diesem Kompetenzerwerb soll die Lehrveranstaltung "Innovationsprojekt" die Studierenden unterstützen. In diesem Zusammenhang sind Design Thinking, Digitalisierung und Diversity Management auf zwei Ebenen Bestandteil der Lehrveranstaltung "Innovationsprojekt: Digitalisierung und Diversity Management": (1) um die Kompetenzen der Studierenden weiterzuentwickeln und (2) als Bestandteil der fachlich zu erarbeitenden Lösung.

(1) Fachkompetenz: Die Studierenden erwerben Kenntnisse grundlegender Konzepte sowie fachspezifischer Wissensbestände der Themen Design Thinking, Digitalisierung und Diversity Management.

Methodenkompetenz: Die Studierenden erwerben Fähigkeiten zur Anwendung von Problemlösungstechniken, insbesondere dem Design Thinking Prozess als Problemlösungsprozess sowie die Fähigkeit zur Anwendung von digitalen Lösungen im Sinne einer digitalen Kompetenz.

Persönliche Kompetenz: Die Studierenden erwerben Fähigkeiten zur Selbstreflexion in Bezug auf die eigene Diversitätsidentität sowie eigene diversitätsbezogene Denk- und Handlungsmuster.

Sozialkompetenz: Die Studierenden erwerben Fähigkeiten, die für die Gestaltung sozialer Interaktionen nützlich oder notwendig sein können, insbesondere dem Zusammenarbeiten in diversen, multidisziplinären Teams.

(2) Bestandteil der Lösung: Im Rahmen der Lehrveranstaltung wird in Kleingruppen ein gesamter Design Thinking-Prozess von einer realen Problemstellung bis zur nutzer_innenzentrierten Innovation durchlaufen. Die Problemstellung sowie der Lösungsbereich fokussieren dabei auf die Themen Digitalisierung und Diversity, konkret 
sollen innovative Ideen zur digitalen Unterstützung von Vielfalt an der Beuth Hochschule für Technik entwickelt werden.

\subsection{Modulbeschreibung}

Bei der Lehrveranstaltung "Innovationsprojekt: Digitalisierung und Diversity Management" handelt es sich um eine fachbereichsübergreifende Lehrveranstaltung, die sowohl von Bachelor- als auch Masterstudierenden besucht werden kann und spricht dadurch eine diverse Studierendenschaft an.

Eine Auseinandersetzung mit dem Thema Diversität soll durch eine aktive Zusammenarbeit über Fachdisziplinen hinweg (Lehrform "Projektarbeit in Kleingruppen") bewusst unterstützt werden. Explizite Bezüge zu den Themen Design Thinking, Digitalisierung und Diversity Management finden sich in dem Lehrziel. Die Studierenden sollen mit Hilfe des Design Thinking Prozesses eine Problemstellung zum Thema Digitalisierung und Diversity bearbeiten. Des Weiteren sieht das Konzept dieser Lehrveranstaltung eine Gruppenbewertung als Prüfungsform vor. Die Zusammenarbeit in divers zusammengesetzten Teams, ein elementarer Bestandteil im Design Thinking, soll belohnt werden und nicht das Bessersein in einer Einzelarbeit, welche die Studierenden über Jahre hinweg auf einen Konkurrenzmodus konditioniert hat (Weinberg 2015, S. 163). Einen Auszug aus der Modulbeschreibung findet sich in Tabelle 1 .

Tabelle 1: Modulbeschreibung

\begin{tabular}{|c|c|}
\hline Datenfeld & Erklärung \\
\hline Titel & Innovationsprojekt: Digitalisierung und Diversity Management \\
\hline Leistungspunkte & $2,5 \mathrm{LP}$ \\
\hline Workload & 2 SWS Ü \\
\hline Lerngebiet & Allgemeinwissenschaftliche Ergänzungen \\
\hline Lernziele / Kompetenzen & $\begin{array}{l}\text { Die Studierenden lernen ein „Innovations-Mindset“ am Beispiel von } \\
\text { Design Thinking kennen und können die einzelnen Prozessschritte } \\
\text { selbstständig anwenden. Dafür wird in Kleingruppen ein gesamter } \\
\text { Innovations-Prozess von einer realen Problemstellung (zum Thema } \\
\text { Digitalisierung und Diversity) bis zur nutzerzentrierten Innovation } \\
\text { durchlaufen. Am Ende der Veranstaltung werden die Studierenden im } \\
\text { Team kreative Lösungen präsentieren können. }\end{array}$ \\
\hline Voraussetzungen & keine \\
\hline Niveaustufe & Bachelor- und Masterstudiengänge \\
\hline Lehrform & $\begin{array}{l}\text { Übung. Die Lehrveranstaltung umfasst Inputs durch die Dozentin und } \\
\text { Projektarbeit in Kleingruppen. }\end{array}$ \\
\hline Status & Wahlpflichtmodul \\
\hline
\end{tabular}




\begin{tabular}{|l|l|}
\hline Häufigkeit des Angebotes & jedes Semester \\
\hline Prüfungsform & $\begin{array}{l}\text { Mündliche Präsentation und ca. 15-seitige schriftliche Ausarbeitung in } \\
\text { Kleingruppe }\end{array}$ \\
\hline Ermittlung der Modulnote & $\begin{array}{l}\text { Die Note ist eine Teamnote und setzt sich aus 50\% mündlicher } \\
\text { Präsentation und 50\% schriftlicher Ausarbeitung zusammen }\end{array}$ \\
\hline Inhalte & $\begin{array}{l}\text { - Sechs Schritte von Design Thinking als Innovationsmethode verstehen } \\
\text { - Bedeutung von Teams und Kollaboration - Gibt es eine neue Wir- } \\
\text { Intelligenz? } \\
\text { - Nutzerbedürnisse analysieren } \\
\text { - Diverse Brainstorming-Methoden anwenden } \\
\text { - Ideen entwickeln und auswählen } \\
\text { - Prototypen erstellen und testen } \\
\text { - Pitches und Präsentationen von Innovationen erlernen }\end{array}$ \\
\hline Literatur & $\begin{array}{l}\text { Brown, T. (2009). Change by Design - How Design Thinking } \\
\text { Transforms Organizations and Inspires Innovation. New York, Harper } \\
\text { Business. } \\
\text { Schmiedgen, J. et al. (2015). Parts Without a Whole? - The Current State } \\
\text { of Design Thinking Practice in Organizations (Study Report No. 97). } \\
\text { Potsdam, Hasso-Plattner-Institut für Softwaresystemtechnik an der } \\
\text { Universität Potsdam. } \\
\text { Uebernickel, F. et al. (2015). Design Thinking - Das Handbuch. } \\
\text { Frankfurt, Frankfurter Allgemeine Buch. } \\
\text { Weinberg, U. (2015). Network Thinking - Was kommt nach dem } \\
\text { Brockhaus-Denken? Hamburg, Murmann Verlag. } \\
\text { Weitere Literatur wird in der Lehrveranstaltung bekannt gegeben. }\end{array}$ \\
\hline
\end{tabular}

\subsection{Kreative Umgebung ("Place")}

Beim kreativen Arbeiten ist der Arbeitsplatz ein wichtiges Grundelement (siehe Kapitel 2.1). Dabei sind nicht unbedingt spezielle Räume oder Möbel notwendig. Auch die üblichen Lehrräume an Hochschulen können so umgestaltet werden, dass ein kreatives und kollaboratives Arbeiten unterstützt wird. Je nach Arbeitsmodi werden dafür ein "Input-Bereich" und verschiedene "TeamarbeitsBereiche" benötigt. Wichtig ist die Möglichkeit die Tische im Raum zu Gruppentischen verschieben zu können sowie Zugriff auf tragbare Metaplanwände oder Whiteboards zu haben. In Abbildung 3 ist der Aufbau eines kollaborativen und kreativen Lern- und Arbeitsumfeldes in einem Lehrraum exemplarisch dargestellt. 

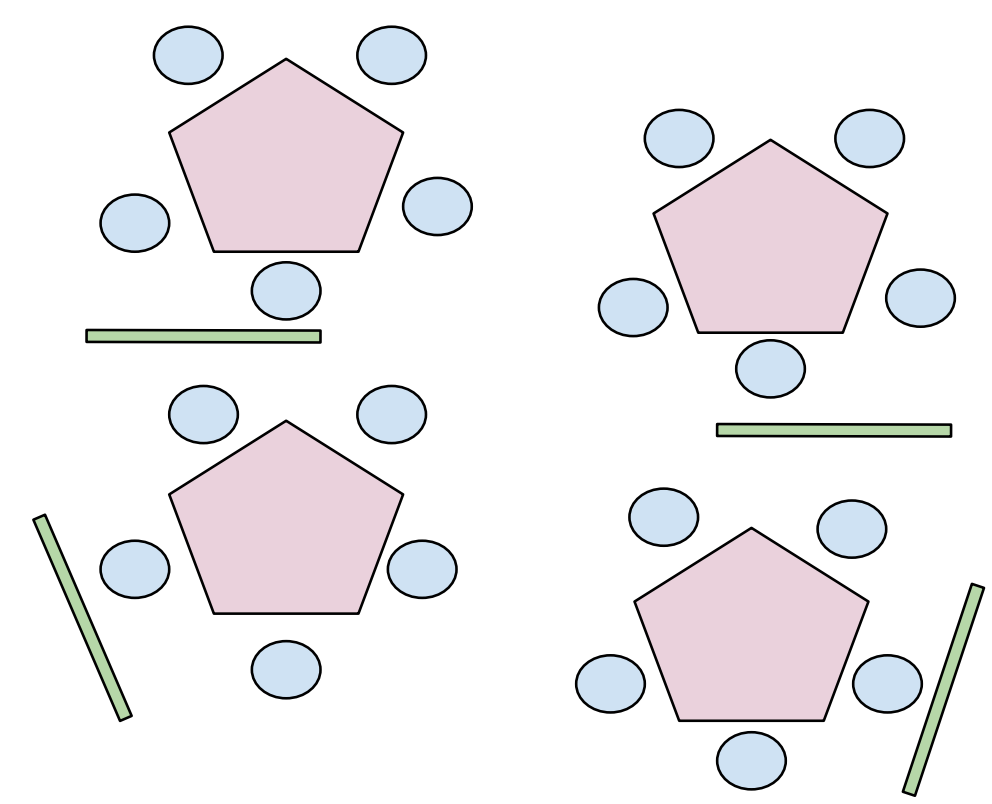

TeamarbeitsBereiche

Input-Bereich

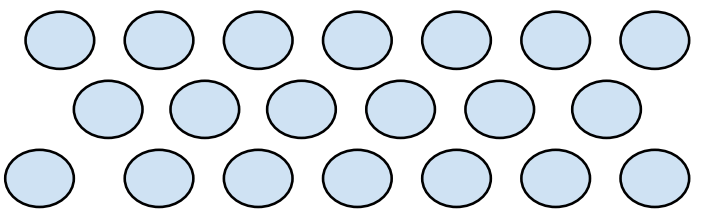

Abbildung 3: Design Thinking Lehrraum

(Quelle: Eigene Darstellung)

Ein kreatives Umfeld zeichnet sich weiterhin durch eine einladende Atmosphäre aus, dazu gehört eine gute Ausstattung der Teamarbeitsplätze mit Arbeitsmaterialien. Nachfolgend wird eine exemplarische Auflistung für vier Kleingruppen à fünf Studierende dargestellt:

- Packpapierrolle mit 50 Blatt (Brownpaper)

- 8 Pakete Post-Its (76x127mm)

- 20 schwarze Stabilo Pens

- 12 Flipchart-Marker

- 120 bunte Klebepunkte

- 4 Scheren

- 1 Tacker

- 1 Cutter Messer

- 4 Klebestifte

- 2 Rollen Kreppband

- 2 Rollen Klebefilm durchsichtig

- 40x buntes Tonzeichenpapier

- 20x feste Pappe

- 100x weißes A4-Papier

- Stoffzuschnitte

- 1 Rolle Alufolie

- 1 Rolle Frischhaltefolie

- Weiteres Material zum Prototypen bauen: Wäscheklammern, Trinkhalme, Luftballons, Pfeifenreiniger, Büroklammern, Holzspieße, Knete, Wolle, Pappbecher, Lego 
Hinzu kommen ein Time Timer (eine spezielle Tischuhr) und ein Gong, um den kreativen Flow zu strukturieren. Dies alles fördert das effektive Arbeiten in Kleingruppen ebenso wie den kreativen Austausch von Ideen, das Entwickeln von Prototypen und die Präsentation von innovativen Lösungsansätzen.

\subsection{Merkmale der Studierenden ("People")}

Die Lehrveranstaltung "Innovationsprojekt" an der Beuth Hochschule für Technik Berlin kann sowohl von Bachelor- als auch Masterstudierenden aller Fachbereiche besucht werden und spricht dadurch eine multidisziplinäre Studierendenschaft mit verschiedenen Erfahrungs- und Wissensständen an. Eine Auseinandersetzung mit dem Thema Diversität kann demnach durch eine aktive Zusammenarbeit über Fachdisziplinen hinweg gezielt unterstützt werden und eine kollaborative Innovationskultur gefördert werden.

Pro Semester nehmen an einer Lehrveranstaltung circa 20 Studierende teil, welche in vier Kleingruppen à fünf Teammitgliedern zusammenarbeiten. Um bewusst eine Zusammenarbeit über Diversitätsgrenzen hinweg zu fördern, wird darauf geachtet, dass jedes Team multidisziplinär und wenn möglich kulturdivers, alters- und geschlechtsheterogen zusammengesetzt ist. Tabelle 2 fasst die verschiedenen Merkmale der Studierenden überblicksartig zusammen:

Tabelle 2: Merkmale der Studierenden

\begin{tabular}{|c|c|}
\hline & Sommersemester 2017 \\
\hline Anzahl der Studierenden & 19 \\
\hline $\begin{array}{ll}\text { Geschlecht: männlich } \\
\text { weiblich }\end{array}$ & $\begin{array}{c}12 \\
7\end{array}$ \\
\hline Art des Studium & Bachelor und Master \\
\hline Besuchter Studiengang* & $\begin{array}{ll}\text { - } & \text { Architektur } \\
\text { - } & \text { Betriebswirtschaftslehre } \\
\text { - } & \text { Druck- und Medientechnik } \\
\text { - } & \text { Medieninformatik } \\
\text { - } & \text { Physikalische Technik/ Medizinphysik } \\
\text { - } & \text { Wirtschaftsingenieurwesen/ Maschinenbau }\end{array}$ \\
\hline $\begin{array}{l}\text { Kultureller Hintergrund der } \\
\text { Studierenden (Selbstauskunft) }\end{array}$ & Deutsch, Türkisch, Arabisch \\
\hline Alter & 18-26 Jahre \\
\hline
\end{tabular}

* Einige Studierende besuchen den gleichen Studiengang.

\subsection{Vorgehensweise und Ablauf der Lehrveranstaltung ("Process")}

Der Ablauf der Lehrveranstaltung folgt in seiner Struktur dem Design Thinking Prozess. Es gibt verschiedene Vorgehensmodelle für Design Thinking. Die hier beschriebene Lehrveranstaltung orientiert sich an den sechsphasigen Prozess des Hasso-Plattner-Instituts (siehe Kapitel 2.1). Am ersten Termin lernen die Studierenden die Methode sowie die Design Thinking Challenge (Problemdefinition) kennen. An den weiteren Terminen wird jeweils eine Phase des Prozesses bearbeitet, angefangen mit dem Verstehen der Design Thinking Challenge die bearbeitet werden soll bis hin zum Testen der entwickelten Prototypen. Am letzten Termin finden die 
Abschlusspräsentationen der Lösungen statt. In den nachfolgenden Abschnitten wird eine Übersicht über die Inhalte der einzelnen Termine gegeben.

\subsubsection{Problemdefinition (Design Thinking Challenge)}

Die Problemdefinition, auch Design Thinking Challenge genannt, ist ein fundamentaler Bestandteil jedes Design Thinking Prozesses. Bei der Problemdefinition handelt es sich um den durch eine Innovation zu erfüllenden Auftrag, ohne Lösungsansätze zu implizieren. Aufgrund dessen ist das Problemfeld neutral zu beschreiben und darf keine Voreingenommenheit enthalten, welche das Innovationsergebnis vorweg beeinflussen könnte (Gürtler \& Meyer 2016, S. 37). Zudem ist der Problembereich zunächst möglichst offen zu gestalten, um das Suchfeld für potentielle Lösungen nicht von Beginn an einzuschränken (Uebernickel et al. 2015, S. 26). In der Regel grenzt die Problemstellung lediglich das Innovationsobjekt, die Zielgruppe/n und den Kontext ein (ebd.). Demnach sind folgende Formulierungen typischerweise Bestandteil jeder Problemdefinition:

Gestalte $\quad$ (Innovationsobjekt / Erlebnis)
für
in / bei / an_linpe / Nutzer/in)
(Kontext / Situation)

Als Design Thinking Challenge für die Lehrveranstaltung "Innovationsprojekt" wurde die Problemdefinition "Gestalte ein neues Studiererlebnis für Geflüchtete an der Beuth Hochschule für Technik Berlin” gewählt. Das Innovationsergebnis soll dabei unter Berücksichtigung des Themas Digitalisierung erarbeitet werden indem eine digitale Unterstützung für ein spezifisches Diversitätsfeld, nämlich studierende Geflüchtete, gesucht wird.

\subsubsection{Phase Verstehen}

Die erste Phase des sechsstufigen Design Thinking-Prozesses heißt "Verstehen" und ist damit der erste Schritt um den Problembereich besser zu durchdringen. Dieser Teil des Problembereichs wird mittels verschiedener Techniken erschlossen (u.a. Semantische Analyse, Mind Map, Charrette), indem Bedürfnisse und Probleme bezüglich der formulierten Design Thinking Challenge ermittelt werden (HPI 2018a). Ziel ist es im Team ein gemeinsames Verständnis der Design Thinking Challenge zu erlangen und Annahmen bezüglicher verschiedener menschlicher Bedürfnisse explizit zu machen. Die Probleme und Bedürfnisse sollen für jedes Teammitglied begreifbar gemacht werden (Blatt \& Sauvonnet 2017, S. 29).

\subsubsection{Phase Beobachten}

In der zweiten Phase des Problembereichs, "Beobachten", wird Empathie für die Zielgruppe aufgebaut (Blatt \& Sauvonnet 2017, S. 30). Dabei werden Techniken der qualitativen Sozialforschung wie Interviews, Beobachten oder Immersion, das Hineinversetzen in den/die Nutzer/in, angewendet (HPI 2018a). Es handelt sich um eine Phase des Informationen Sammelns, Lernens und Erforschens (Gürtler \& Meyer 2016, S. 43). Ziel ist es Bedürfnisse von Kund_innen, Nutzer_innen und weiteren Stakeholdern zu identifizieren (Uebernickel et al. 2015, S. 27). Ü̈ber deren erzählte Erlebnisse und Gefühle soll versucht werden Empathie zu entwickeln und sich mehr und mehr "in die Rolle des potentiellen Nutzers versetzen zu können, dessen Denkweise nachvollziehen zu können und seine eigentlichen Bedürfnisse zu verstehen" (Gürtler \& Meyer 2016, S. 40).

In dieser Lehrveranstaltung wurden Interviews mit Geflüchteten sowie mit Beteiligten des Projektes „Refugees Welcome“ an der Beuth Hochschule für Technik Berlin geführt. 


\subsubsection{Phase Sichtweise definieren}

Ziel der dritten Phase "Sichtweise definieren" ist es, durch eine Synthese der gewonnenen Informationen aus der Recherchephase einen gemeinsamen Standpunkt (Point of View (POV)) zu definieren (Blatt \& Sauvonnet 2017, S. 32). Zunächst geht es darum, die Vielzahl an Informationen zusammenzutragen und zu strukturieren, um diese anschließend zu verdichten (Gürtler \& Meyer 2016, S. 44). Durch diese Art der Synthese, also das strukturierte Gewichten und Filtern der gesammelten Informationen wird die Fragestellung in eine lösbare Problemaussage umstrukturiert (HPI 2018a). Als Techniken sind die Persona, also die Beschreibung eines_r typischen Nutzer_in (Gürtler \& Meyer 2016, S. 48) und Costumer Journey Map oder Storyboard, das Wiedergeben der entstandenen Informationen in einer Story zu nennen (Blatt \& Sauvonnet 2017, S. 32f.). Das Ergebnis der Synthese wird in der Regel als "Point of View" zusammengefasst und besteht aus einer oder mehrerer Personae, deren Bedürfnissen und den wichtigsten Erkenntnissen aus der Recherchephase. Eine typische Formulierung eines POV ist häufig wie folgt strukturiert:

- Wir haben getroffen

- Wir waren inspiriert zu entdecken

- Wir möchten ihm/ihr dabei helfen

\subsubsection{Phase Ideen finden}

Die Phase "Ideen finden" ist die vierte im Gesamtprozess und die erste Phase im Lösungsbereich. Ausgehend von dem entwickelten Standpunkt (POV) werden in dieser Phase Ideen generiert (Uebernickel et al. 2015, S. 30). Dabei ist jede Idee oder die Verknüpfung mehrerer Ideen als potentielle Problemlösung anzusehen. In dieser Phase geht es zunächst um das quantitative Generieren von Ideen und anschließend um deren Evaluierung (HPI 2018a). Die zentrale Technik der Ideenfindungsphase ist das Brainstorming mit einer Vielzahl an verschiedenen Ausprägungen, u.a. Perspektivwechsel (z.B. „Was würde Superman machen?“), Bodystorming (im Kreis laufen und jede Runde eine Idee aufschreiben) oder das klassische stille Brainstorming, bei dem jede_r für sich Ideen aufschreibt (Gürtler \& Meyer 2016, S. 51ff.). Die Evaluierung der verschiedenen Ideen kann mithilfe von Clustern erfolgen (Blatt \& Sauvonnet 2017, S. 35 f.) bei der verschiedene Kriterien zur Ideenauswahl angelegt werden können, beispielsweise:

- Was ist die wildeste/radikalste Idee?

- Welche Idee scheint am einfachsten zu realisieren?

- Welche Idee ist für den_die Nutzer_in am besten?

\subsubsection{Phase Prototypen bauen}

Die fünfte Phase "Prototypen bauen" hat zum Ziel, die entwickelten Ideen für echte Produkte, Dienstleistungen oder Geschäftsmodelle in Form von Prototypen zu simulieren und erlebbar zu machen (Uebernickel et al. 2015, S. 31). Konkret geht es darum, die Idee oder bestimmte Aspekte der Idee zu veranschaulichen, zu konkretisieren und anfassbar zu machen, indem möglichst schnell und einfach erste Prototypen gebaut werden (Gürtler \& Meyer 2016, S. 55). Im Team fördert die Erstellung von Prototypen kreative sowie produktive Erfahrungen und ist ein Mittel um die jeweiligen Gedanken zu verknüpfen (HPI 2018a) und kann auch als Kommunikationsmedium im Team dienen (Uebernickel et al. 2015, S. 31). Dabei können die Prototypen in Form einer Skizze umgesetzt werden, als LEGO-Welt, aus Pappe oder buntem Papier oder auch als Rollenspiel, beispielsweise wenn es um eine Dienstleistungsidee geht (Gürtler \& Meyer 2016, S. 55). 


\subsubsection{Phase Testen}

Im Anschluss an die Erstellung der Prototypen geht es in der sechsten und letzten Phase um das "Testen" dieser Prototypen. Dafür werden die Prototypen mit potentiellen Nutzer_innen getestet, um praxisnahes Feedback zu erhalten (Blatt \& Sauvonnet 2017, S. 39). Da ein Prototyp das aktuelle Wissen sowie Annahmen des Status quo repräsentiert, zeigt diese Phase die Notwendigkeit für das iterative Vorgehen im Design Thinking Prozess. Das Team ist dazu angehalten mehrmals zu testen und den Prototypen entsprechend des Feedbacks weiterzuentwickeln (Gürtler \& Meyer 2016, S. 58; HPI 2018a). Möglicherweise gibt es durch das Feedback von Testenden sogar neue Ideen oder hilft dem Team den Problem- und Lösungsraum noch besser zu verstehen (Uebernickel et al. 2015, S. 34).

\subsubsection{Abschlusspräsentationen}

Nachdem die sechs Phasen des Design Thinking Prozesses durchlaufen wurden finden am letzten Sitzungstermin der Lehrveranstaltung "Innovationsprojekt" die Abschlusspräsentationen statt. In Kleingruppen werden sowohl die einzelnen Schritte des Design Thinking Prozesses als auch die nutzerzentrierte Innovation in Form eines Prototypen präsentiert. Die Präsentation ist dabei meist gekennzeichnet durch einen Methodenmix aus Powerpoint-Präsentation, Flipcharts und einem Rollenspiel.

Wie aus der obigen Modulbeschreibung (vgl. Tabelle 1: Modulbeschreibung) ersichtlich, umfasst die Prüfungsleistung für das Modul zwei Elemente, die die Note zu je $50 \%$ bestimmen: (1) die beschriebene Abschlusspräsentation und (2) eine ca. 15-seitige schriftliche Ausarbeitung in den Teams. Dabei werden beide Prüfungsleistungen bewusst so ausgelegt, dass die gemeinsame Leistung als Team entscheidend ist und als solche benotet wird. Denn nicht nur die heutige Arbeitswelt im Allgemeinen ist gekennzeichnet durch eine zunehmende Teamarbeit, sondern auch Design Thinking im Speziellen ist stets eine Teamleistung.

\section{Ergebnisse: Innovative Ideen zur digitalen Unterstützung von Vielfalt an der Beuth Hochschule für Technik}

Im Folgenden werden vier studentische Essays vorgestellt, welche die Studierenden über ihre innovativen Ideen zu den Themen Digitalisierung und Diversity Management erstellt haben. Alle Ideen wurde mithilfe des Design Thinking Prozesses von den Studierenden erarbeitet und folgten dem in Kapitel 3.5 beschrieben Vorgehen im Modul "Innovationsprojekt: Digitalisierung und Diversity Management" an der Beuth Hochschule für Technik Berlin.

Da sich die von den Studierenden in den ca. 15-seitigen Essays ebenfalls dargestellten Prozessschritte - Phase Verstehen bis Phase Testen (vgl. Kapitel 3.5) - untereinander und mit den obigen Ausführungen inhaltlich überschneiden bzw. wiederholen, umfassen die folgenden Unterkapitel lediglich die Ergebnisdarstellung aus vier studentischen Essays in einer leicht geglätteten Version, um einen besseren Lesefluss zu bieten. Dabei wurden bewusst keine größeren Änderungen oder Ergänzungen vorgenommen, weil die Essays sehr gut widerspiegeln, in welcher Tiefe die Studierenden das bearbeitete Thema durch die Methode verstanden und durchdrungen haben. Zusätzlich werden dadurch die jeweiligen Meinungen, Wahrnehmungen und Interpretation aus Sicht der Studierenden deutlich und gerade im Design Thinking ist eine derartige subjektive Einschätzung oder ein Gefühl für die Nutzer_innen essentiell für die Entwicklung von nutzer_innenzentrierten Innovationen. 
Im Anschluss an die der vier Ergebnisdarstellungen werden diese in Kapitel 5 in Bezug auf die von den Studierenden entwickelten Kompetenzen und in Bezug auf die inhaltlichen Lösungen reflektiert.

\subsection{Website „Refugees Reiter“}

Joyce Jesch, Felix Pöhle, Robert Hauser, Andreas Graf, Holger Motz

Aus den Ergebnissen der Phasen 1 bis 6 des Design Thinking Prozesses wurde der Prototyp „Refugees Reiter" entwickelt, welches die größte Einstiegshürde für geflüchtete Studieninteressierte, nämlich den Zugang zu relevanten Informationen, lösen soll. In den Phasen 1 bis 3 konnte als Hauptproblematik identifiziert werden, dass Geflüchtete, welche mit den deutschen Hochschul- und Organisationsstrukturen nicht vertraut sind und darüber hinaus Sprachbarrieren zu bewältigen haben, auf eine vereinfachte Bedienbarkeit angewiesen sind. Den Geflüchteten sollen die für sie passenden und relevanten Informationen möglichst niedrigschwellig zugänglich gemacht werden.

Auf der Beuth-Homepage erscheinen wichtige Studien- und Kursinhalte versteckt und sind nur über Mehrfach-Clicks zu erreichen. Zudem sind sie für nicht-deutschsprachige Personen nur schwer verständlich. Einige Studiengänge sowie deren Studien- und Kursinhalte sind außerhalb Deutschlands atypisch, Fachwörter und Begriffe können nicht zuverlässig und adäquat in andere Sprachen übersetzt werden. Die Studien- und Kursinhalte sind in unterschiedlichen Untermenüs zu finden und folgen zumeist etlichen Links und Querverweisen. Ziel des Prozesses war es, alle für den Bewerbungsprozess bzw. ein Studium benötigten Informationen für Geflüchtete kompakt, übersichtlich und strukturiert zur Verfügung zu stellen und den Nutzer_innen das Gefühl zu vermitteln: „Hier bin ich richtig.“ Da die Beuth-Homepage die erste Anlaufstelle für den Großteil der Studieninteressierten darstellt, war dies der offensichtliche Punkt anzusetzen und die Startseite der Beuth-Homepage um einen eigenen Refugees-Reiter zu ergänzen.

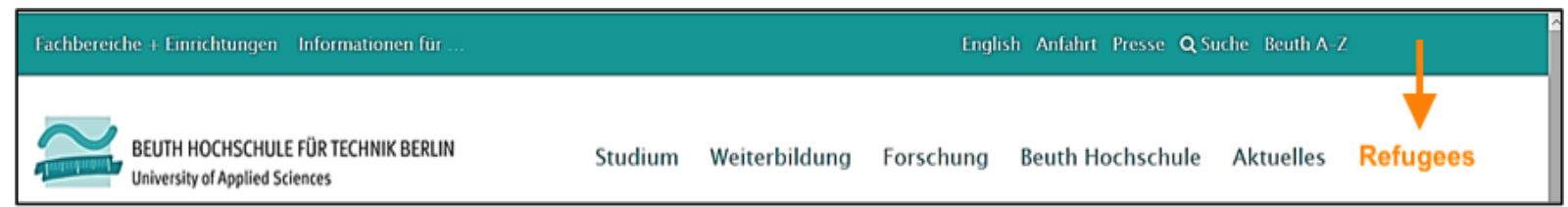

\section{Abbildung 4: Prototyp Refugees-Reiter - zusätzlicher Reiter auf der Beuth-Homepage}

(Quelle: https://www.beuth-hochschule.de/ (29.06.2017))

Dieser Reiter beinhaltet ein neu gestaltetes, einfaches und übersichtliches Menü. Alle notwendigen Informationen befinden sich, je nach Informationswunsch, hinter großen logisch angeordneten Buttons in der Mitte der Seite bzw. des Frames. In Abbildung 5 sind beispielsweise Schaltflächen für Bachelor, Master und Bewerbung zu sehen. 


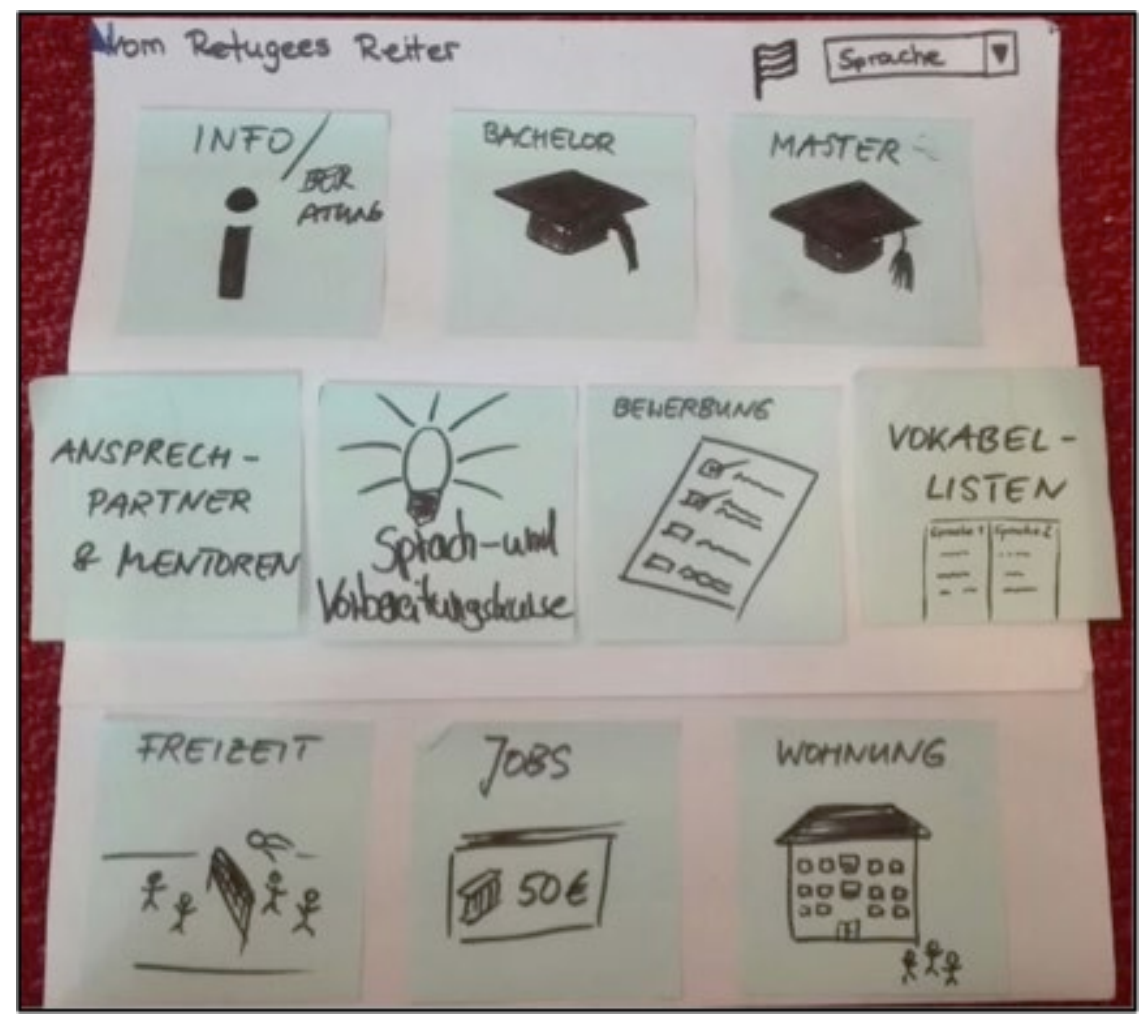

\section{Abbildung 5: Prototyp der Startseite des "Refugees Reiters"}

(Quelle: Eigene Darstellung)

Die Verständlichkeit soll zum einen durch eine eindeutige und einfache Beschriftung und zum anderen durch unterstützende Piktogramme gewährleistet werden. Zusätzlich kann über ein Dropdown-Menü die Sprache ausgewählt werden. Zu Beginn sind neben Deutsch, Englisch und Arabisch auch „einfaches Deutsch“ angedacht, um schwierige Erklärungen, beispielsweise bei Erklärungen zum Bewerbungsprozess, zu simplifizieren. Jeder der in Abbildung 5 abgebildeten Menüpunkte enthält weitere Buttons mit immer tiefergehenden und aufgearbeiteten Informationen zum jeweiligen Themengebiet. Die tiefergehende Strukturierung der Informationen ist nach Thema zwar individuell gestaltbar, jedoch soll sich die Ausgestaltung an Gesamtdesign und -struktur orientieren, um die Übersichtlichkeit und Einfachheit zu wahren. Bei der Auswahl des Buttons „Bachelor“ beispielsweise, werden alle Bachelorstudiengänge angezeigt.

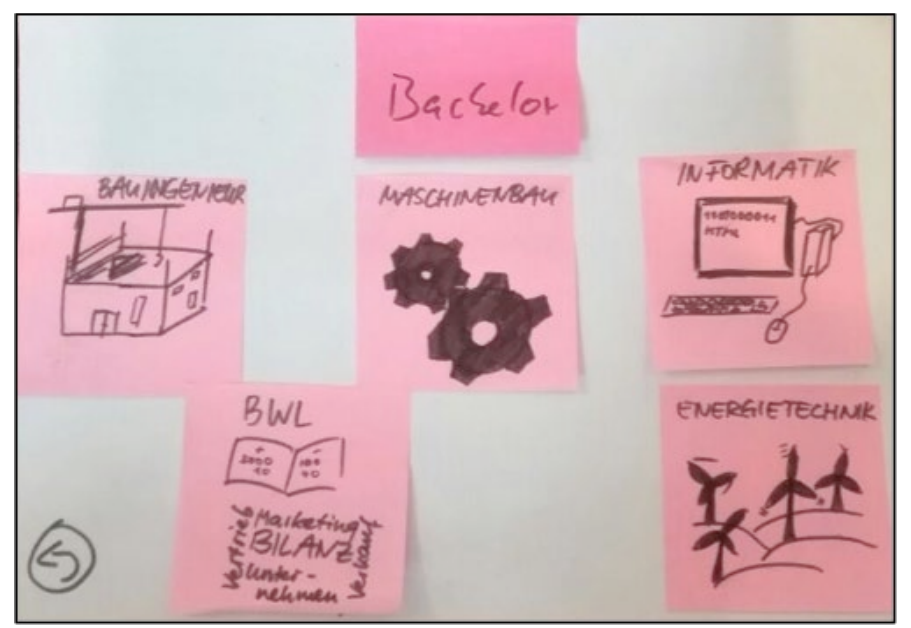

Abbildung 6: Darstellung der Studiengangsauswahl

(Quelle: Eigene Darstellung) 
Daraufhin kann der gewünschte Studiengang ausgewählt werden, woraufhin die Studieninhalte nach Semestern sortiert angezeigt werden. Bei Semesterauswahl werden die Semestermodule und nach Auswahl eines Moduls, die Schwerpunkte bzw. deren Inhalte geöffnet. Weiterhin soll nach Modulauswahl bei Bedarf eine Vokabelliste kreiert werden können, um gebräuchliche Fachwörter schnell zur Hand und übersetzt bzw. visuell erklärt zu haben. Dies soll vor allem dem Verständnis zu Studienbeginn dienen und dem Studierenden das Folgen des Stoffes erleichtern. Die Idee dahinter ist es logische Abfolgen zu schaffen, das heißt es geht in der Informationsrangfolge nur höher oder tiefer oder vom Allgemeinen zum Speziellen.

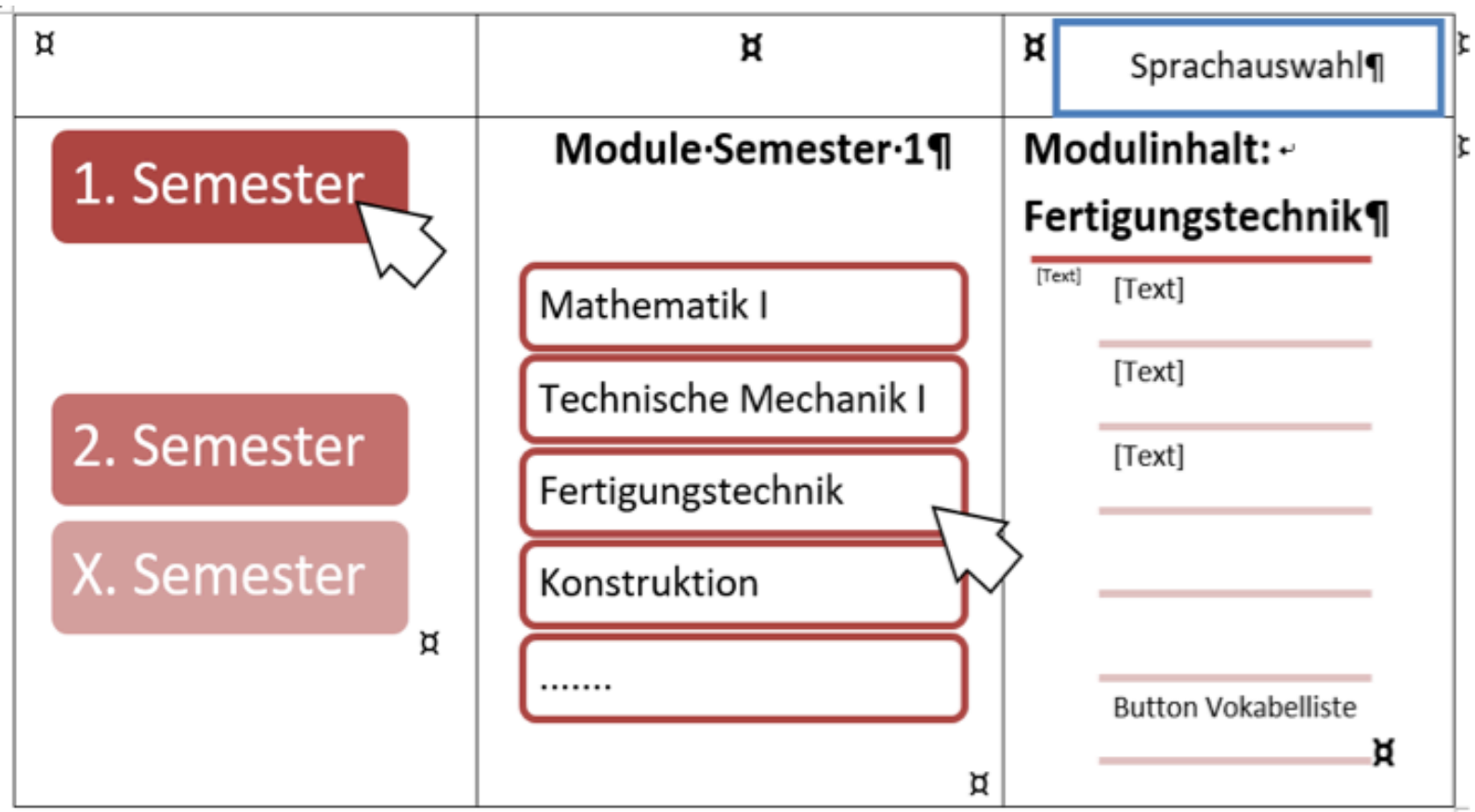

Abbildung 7: Darstellung Untermenü Studiensemester und -inhalte

(Quelle: Eigene Darstellung)

Betrachtet man abschließend die vorausgegangene Problemstellung sowie die Bedürfnisse der zurzeit sehr schweren Informationsbeschaffung für Geflüchtete, so wird deutlich, dass die Lösung "Refugees Reiter" ein nutzer_innenzentrierter Prototyp für ein spezifisches Problem vieler Geflüchteter darstellt. Durch eine strukturierte Vorgehensweise im Design Thinking Prozess, die Bedürfnisse Schritt für Schritt zu identifizieren und aus verschiedenen Blickwinkeln zu betrachten, konnte eine relativ einfache und hilfreiche Lösung generiert werden.

Durch die Einfachheit der Innovation und dessen Effektivität ist die Umsetzung relativ leicht und realitätsnah, da es keiner größeren Änderungen im Web-System der Hochschule bedarf. Der „Refugees"-Reiter könnte beispielsweise im Rahmen eines Hochschulprojektes höheren Semesters von (Medien-)Informatiker_innen semesterbegleitend programmiert und gestaltet werden. Somit könnte mit einer zeitnahen und günstigen Implementierung geflüchteten Studieninteressierten effektiv geholfen werden. Zukünftig könnte dieses System schrittweise weiterentwickelt werden. Es könnte beispielsweise um weitere Sprachen ausgebaut werden. 


\subsection{App „BeutHelp“}

\section{Daniel Buschbacher, Alexander Winterholler, Julius Brett, Ahmed Abdelmula, Thomas Graaf}

Als Ergebnis der Phasen 1 bis 6 des Design Thinking Prozesses wurde die App "BeutHelp" entwickelt. Diese weist sich dadurch als innovativ aus, da wir die Kernprobleme des_der Nutzers_in erkannt haben, wie z.B. die Scheu vor Kontaktaufnahme und die Möglichkeit jederzeit eine Frage zu stellen unabhängig von den Öffnungszeiten des Studieninfoservices. Die entwickelte App stellt eine zentrale Wissensdatenbank mit sämtlichen Anleitungen und Hinweisen für alle Themen dar, die eine geflüchtete Person möglicherweise sucht und benötigt.
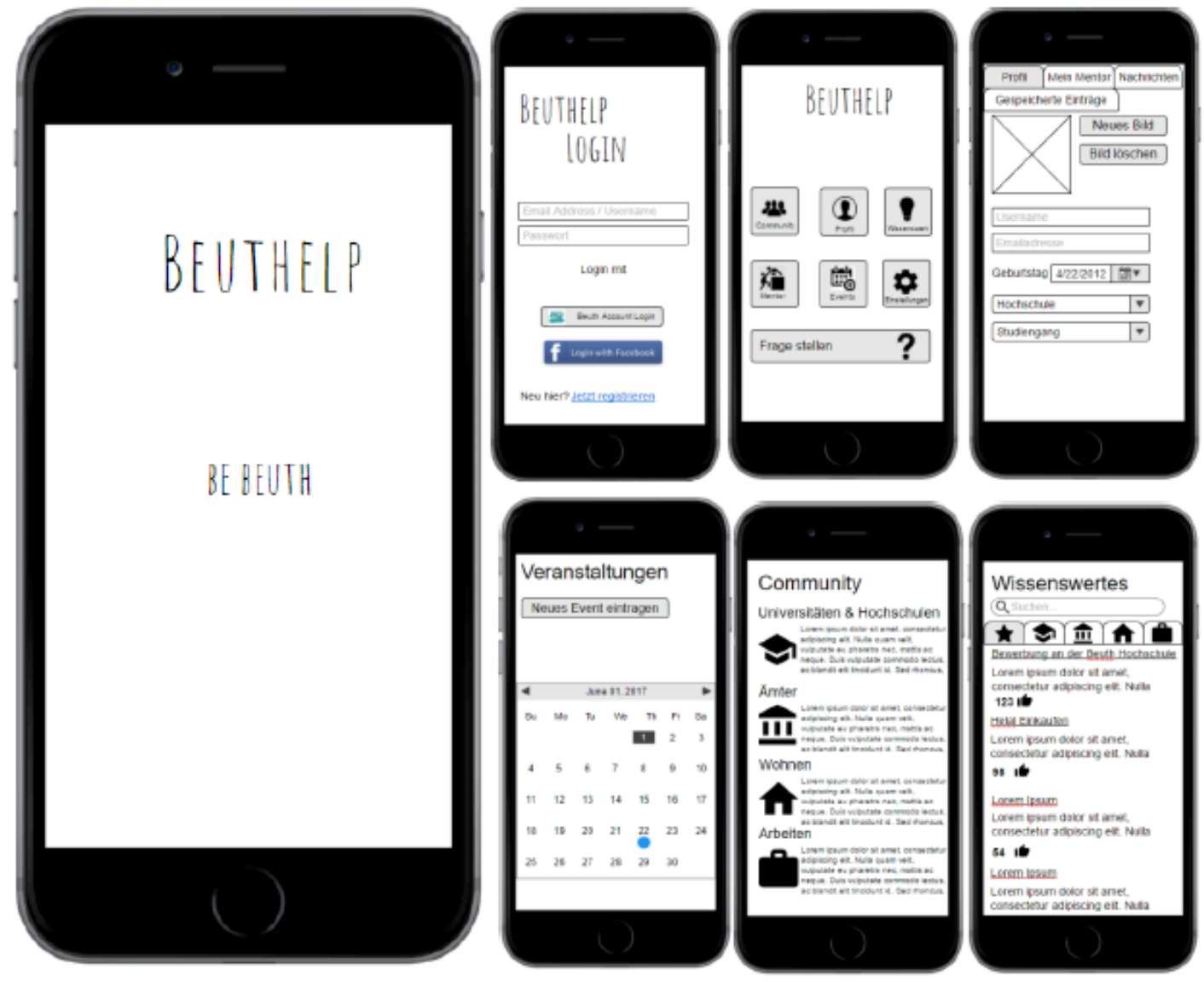

\section{Abbildung 8: Prototyp "BeutHelp"}

(Quelle: Eigene Darstellung)

Die App bietet zwei verschiedene Funktionen: zum einen ein Forum, worüber Diskussionsrunden initiiert werden können und zum anderen ein Eventsystem zur einfachen Veranstaltungsplanung, zu der auch weitere Personen eingeladen werden können. Die App steht dementsprechend auch in anderen Sprachen zur Verfügung, um Geflüchtete, die kaum oder noch kein Deutsch sprechen, zu unterstützen. Das Highlight der App ist das Mentoring-System, in dem immatrikulierte Beuth Studierende sich als Mentor_in registrieren können, um einzelne Geflüchtete zu begleiten. Ziel ist es dabei vor allem die "unsichtbare Wand" zu entfernen, die in der Wahrnehmung von geflüchteten Menschen zwischen ihnen und den "Einheimischen" existiert und die Kontaktaufnahme erschwert. Zudem können Mentoren_innen die Wissensdatenbank mit weiteren Anleitungen ergänzen und Sorge dafür tragen, dass keine falschen Informationen veröffentlicht werden. Durch ein Rating-System wird für den_die Nutzer_in erkennbar, ob ein Post hilfreich ist oder nicht. Sofern Nutzer_innen Benachrichtigungen von der App zulassen, werden sie außerdem über Neuigkeiten informiert, 
unabhängig ob sie die App derzeit benutzen oder nicht; z. B. ob eine gestellte Frage beantwortet, ein erstellter Beitrag im Forum kommentiert oder ob eine neue Nachricht an zugestellt wurde.

Wir konnten unsere innovative Idee nicht nur auf Papier festhalten, sondern tatsächlich auch einen handfesten Prototyp entwickeln (siehe Abbildung 8), um die Idee präsentieren und sogar testen zu können.

Die offene Frage bei der App BeutHelp ist, wie kann die Anwendung baldmöglichst durch Nutzung der vorhandenen Technik an der Beuth Hochschule für Technik Berlin umgesetzt werden? Besteht weiterhin die Motivation für aktive und hilfsbereite Nutzer und Nutzerinnen? Eine Möglichkeit wäre, wenn die Anwendung im Rahmen eines Studium Generale-Projektes weiterentwickelt und eingeführt werden könnte. Zum Beispiel könnten Informatik-Studierende die App ausarbeiten. Druck- und Medientechnik-Studierende könnten eine Konzeption für die App ausarbeiten und Werbung am Campus betreiben, u.a. über die Beuth-Homepage oder mittels Flyer.

\subsection{Digitaler Terminal „BE(uth) Connected“}

\section{Burhan Sahin, Eray Alcinakaya, Gürkan Özcan, Isa Li Pätzold, Lara Lohmann}

Auf Basis der Ausarbeitung der sechs Phasen Verstehen bis Testen des Design Thinking Prozesses, stellen wir nachfolgend unsere Lösungsidee vor. Als besonders hilfreich hat sich das Treffen mit Geflüchteten erwiesen. Dabei hatten wir zwei Mal die Möglichkeit informative Interviews zu führen. In der Beobachtungsphase lernten wir potenzielle Nutzer_innen und ihre Herausforderungen intensiver kennen. Dabei wurde deutlich, dass die sprachlichen Einschränkungen ein entscheidender Punkt sind. In der Testing Phase gaben die Geflüchteten Feedback zu unserem Prototypen. Dieses Vorgehen hat den Entwicklungsprozess sehr gestärkt.

Wir haben unsere Idee greifbar gemacht, indem wir einen dreidimensionalen Prototypen entwickelt haben. Dabei sind wir ganz pragmatisch vorgegangen. Unsere Vorgehensweise zur Planung und Entwicklung unseres Prototyps basiert auf der Minimierung von sprachlichen und kulturellen Restriktionen für die Geflüchteten an der Beuth Hochschule für Technik Berlin. Unser Ziel war es, unsere Idee so gut wie möglich veranschaulichen zu können, sodass unser Leitmotiv schnell und einfach ersichtlich wird. Schließlich soll unsere Innovation jedem_r Nutzer_in eine adäquate und direkt verwertbare Antwort auf Fragen geben oder zumindest einen Schritt weiter helfen. Die wichtigste Funktion unseres digitalen InfoPoint ist die Sprachenvielfalt. 


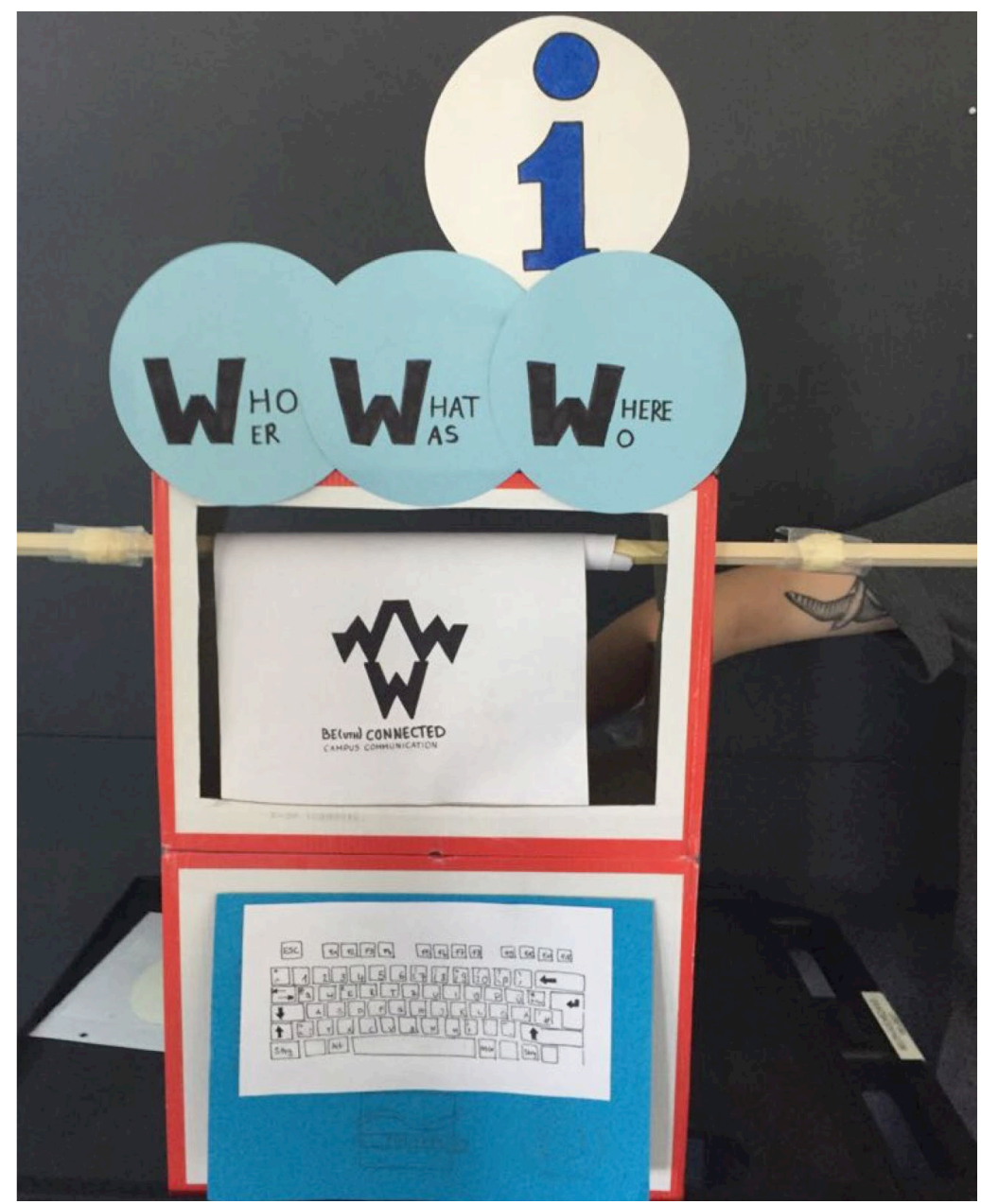

Abbildung 9: Prototyp Who What Where

(Quelle: Eigene Darstellung)

In erster Linie soll eine Sprachbarriere überwunden und keine neuen Probleme verursacht werden, dies wäre z.B. durch eine zu komplizierte Bedienung der Fall. Deshalb steht an oberster Stelle die Fähigkeit unseres technischen Systems, sich durch eine einfache und intuitive Bedienung an die Bedürfnisse der Nutzer_innen anzupassen. Durch eine nutzer_innenfreundliche Navigation können wir die Nutzungsqualität sehr hoch halten. Ziele effektiv, effizient und zufriedenstellend zu erreichen, ist keine Selbstverständlichkeit, vor allem nicht dann, wenn eine Sprachbarriere vorhanden ist. Weil Informationen, die eine bekannte Struktur aufweisen, sehr viel einfacher zu verarbeiten und zu behalten sind, haben wir ein einfaches und ansprechendes Layout für unser technisches System bevorzugt. „Wo bin ich?“, „Was tut diese Seite?“, „Wo bin ich gewesen?“ und „Wohin kann ich gehen?" sind die Fragen, die wir uns gestellt haben, als wir die Navigation für das System entwickelt haben.

Die Navigation der Seite muss so schnell erkennbar sein, da der Nutzende meist ungeduldig ist und schnell an Informationen gelangen möchte. „Wieso?“",Weshalb?“"Warum?“ waren die ersten Fragen,die uns durch unsere Köpfe schossen, als es schließlich um einen passenden Namen für unseren InfoPoint ging. Selbstverständlich musste es ein Name sein, der für jeden verständlich ist, daher die Entscheidung für die englische Übersetzung. „WHO? WHAT? WHERE? (WER? WAS? WO?)“ schien uns der passende Name für unseren digitalen Auskunftsdienst auf dem Campus der Beuth Hochschule für Technik Berlin zu sein. 


\subsection{Datenbank „,TermynI“}

Samira Arifovic, Said Sivük, Tri Nguyen Lam, Christopher Alexander Heiting, Emna Sofiene, Benjamin Förster, Simon Schneider

Als Ergebnis aus den Phasen 1 bis 6 des Design Thinking Prozesses, wurde unsere Datenbank "TermynI" entwickelt. Dieser Prototyp ist auf ein effizientes Lernen an der Hochschule ausgerichtet. Ziel ist es, geflüchteten Studierenden konstruktiv zur Seite zu stehen und sie beim effizienten Lernen zu unterstützen. Der Prototyp konzentriert sich auf eine komplexe Ausarbeitung der fachspezifischen Vokabeln jedes Fachbereiches an der Beuth Hochschule für Technik Berlin, wobei ein organisierter Erweiterungsprozess der dargestellten Vokabelliste unentbehrlich ist.

Eine gute Qualität der sprachlichen Übersetzung der Fachbegriffe hat eine hohe Priorität, sodass ein großer Nutzen für die Geflüchteten entsteht. Die angebotene Dienstleistung seitens Studierender soll kostenfrei erhältlich sein. Die Vokabelliste kommt in jedem Fachbereich zum Einsatz.

Für die Funktionstüchtigkeit des Prototyps müssen die Informationen immer wieder ergänzt werden. Ferner ist das Engagement seitens Studierender der Hochschule für die Erweiterung und Pflege der Datenbank unentbehrlich. Ein Meilenstein für die dauerhafte und kontinuierliche Umsetzung dieses Vorhabens ist die Hochschule, da sie das Budget für ein Smartboard und die dazu notwendigen Räumlichkeiten zur Verfügung stellt. Eine Steigerung zum Prototypen erfolgt unmittelbar durch die Anwendung als App. Die Nutzung der App dient nicht nur der erwähnten Hilfestellung, sondern auch für die Nutzung der Vokabellisten während der einzelnen Vorlesungen.

\begin{tabular}{|c|c|c|c|c|c|}
\hline \multirow{2}{*}{ 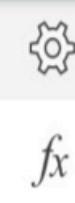 } & \multicolumn{5}{|c|}{ Dämmung } \\
\hline & \multicolumn{5}{|c|}{ Dämmung } \\
\hline$\Delta$ & A & B & c & D & \\
\hline 1 & \multicolumn{5}{|c|}{ Fachbereich III- Fachbegriffe } \\
\hline 2 & & & \multicolumn{3}{|c|}{ Syrien/Somalia/Sudan Afghanistan } \\
\hline 3 & Begriffe & Englisch & Arabisch & Persisch & \\
\hline 4 & Beton & & asmant & 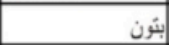 & \\
\hline 5 & Bewehrung & & taeziz & اتقريت & \\
\hline 6 & Bindemittel & & ghalaf & بن & \\
\hline 7 & Brandschutz & & alhimayat min alharay & 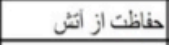 & \\
\hline 8 & Dämmung & & aleuzal & عائ & \\
\hline 9 & Dübel & & watad & بول & \\
\hline 10 & Einfeldträge & & tamtadu wahid alearic & تئ بل دهاتك & \\
\hline 11 & Hochbau & & tashyid almabani & سلاختَو ساز ساختَّان & \\
\hline 12 & Mauerwerk & & masunia & البنابي & \\
\hline 13 & Naturstein & & alhajar altabieiu & سنـ طبيعى & \\
\hline 14 & Stahl & Steel & Sulb & إقلاد & \\
\hline 15 & Stahlbau & & albina' alsulb & لساخترو ساز فو لاد & \\
\hline 16 & Stahlbeton & & alkhrsant almusalaha & 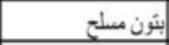 & \\
\hline 17 & & & & & \\
\hline
\end{tabular}

\section{Abbildung 10: Prototyp Vokabelliste als Smartboard}

(Quelle: Eigene Darstellung)

Der Name für unseren Prototyp lautet: „TermynI“. Bei diesem Wort handelt es sich, passenderweise wenn man die Funktion bedenkt, um ein Amalgam - ein Wort aus verschiedenen Wörtern. Das „my“ 
für den Bezug zur Persönlichkeit der Nutzer_innenebene. Eine Endung die wie „Ini“ klingt, da wir die INI-Räume für dieses Projekt verwenden. INI-Räume sind „Initiativ-Räume“ an der Hochschule, die von den Studierenden selbstständig verwaltet werden. INI-Räume sind damit Anlaufstellen für alle Studierenden und bieten neben bequemen Sitzgelegenheiten (Sessel und Couches) meist auch Getränke, Knabberzeug, Kopierer und Drucker. Insgesamt klingt das Wort „TermynI“ wie „Terminal“", ein elektrisches Interface, worum es sich hier ja auch handelt. Des Weiteren bedeutet der Begriff „Termini“ im italienischen „Ort“.

Im Rahmen des Testings wurde „TermynI“ sowohl in der Variante als Smartboard als auch in Form einer App von den anwesenden Testpersonen auf Tauglichkeit geprüft. Die genannten Verbesserungsvorschläge bzw. die neuen Ideen seitens der Tester_innen wurden festgehalten und anschließend von uns umgesetzt. Im Folgenden sind die zu durchlaufenden Schritte am Beispiel der App dargestellt. Die Funktionsweise von „TermynI“ wurde angesichts der genannten Vorschläge verbessert.

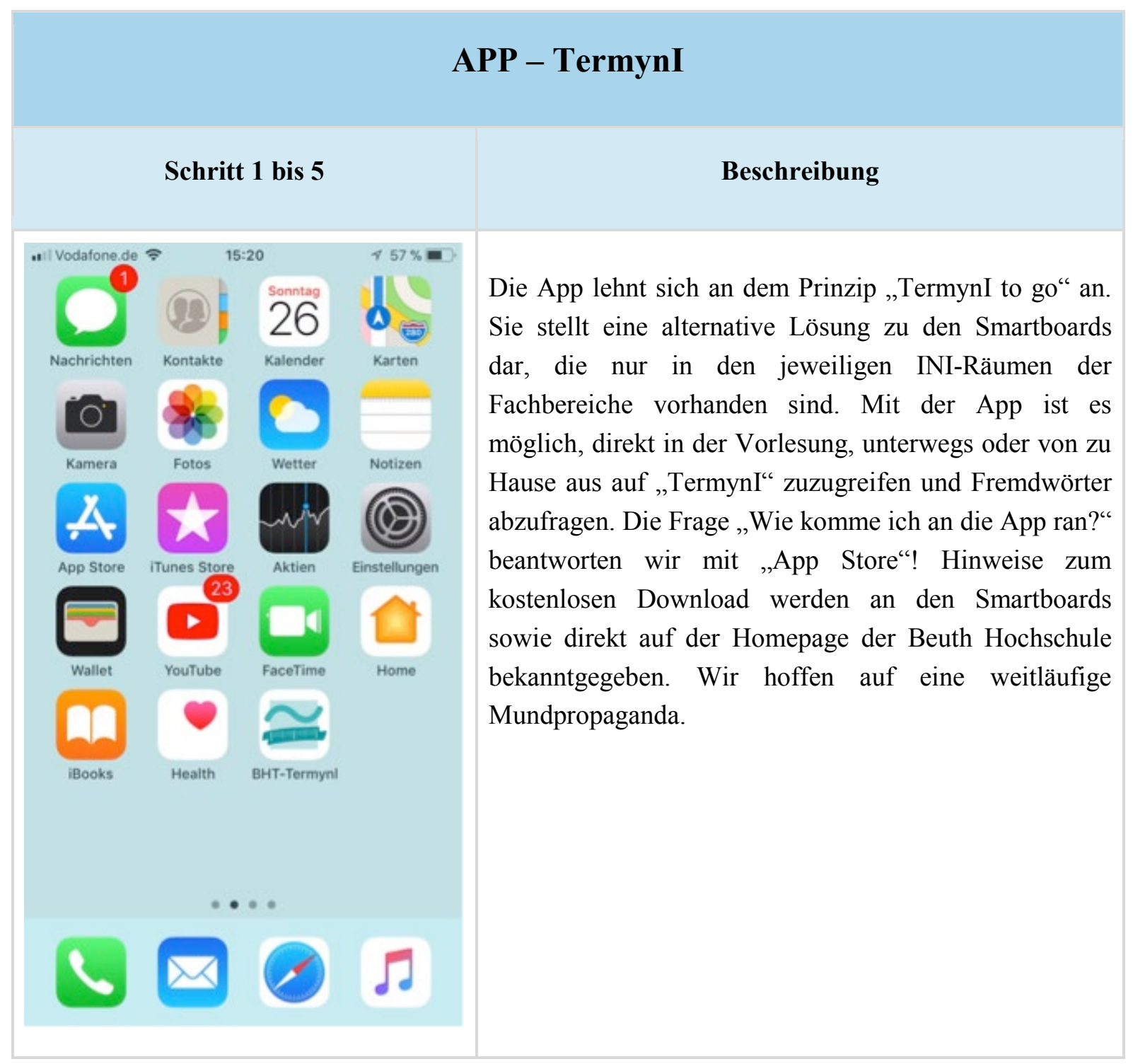


Beuth Hochschule

Fachbereiche

\section{Bitte wählen Sie einen}

Fachbereich aus:

Fachbereich I

Fachbereich II

Fachbereich III

Fachbereich IV

Fachbereich V

Fachbereich VI

Fachbereich VII

Fachbereich VIII
Unser Alleinstellungsmerkmal sind die fachspezifischen Fremdwörterlisten. Studierende können Fremdwörter für ihren Fachbereich anzeigen lassen, ohne von endlosen Listen mit fachfremden Wörtern überhäuft $\mathrm{zu}$ werden. Der_die Nutzer_in öffnet die App und wird folglich gebeten, einen der acht Fachbereiche auszuwählen. Unseren App-Prototypen haben wir durch einleitende Aufforderungssätze ergänzt.

„Wer ist für TermynI zuständig?“ - Hier gilt das Prinzip: Die Nutzer_innen der App sollen selbst beteiligt werden. Der Einsatz studentischer Hilfskräfte wäre darüber hinaus sehr hilfreich.
Beuth Hochschule

$\rightarrow$ Fachbereich III

\section{Bitte wählen Sie ihr}

Heimatland aus:
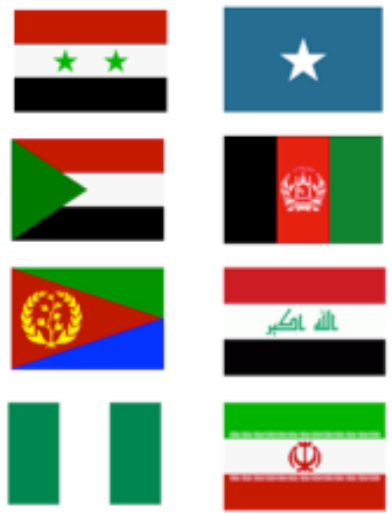

Nachdem der_die Studierende einen Fachbereich ausgewählt hat, wird er_sie aufgefordert, eine der gelisteten Länderflaggen zu bestätigen. Damit wird die Fremdwörtersuche auf eine Sprache reduziert. Die Auswahlfunktion, in Form der Nationalflaggen, ermöglicht eine selbsterklärende, heimatbezogene und einfache Darstellungsvariante. Aus den gelisteten Ländern (u.a. Syrien, Irak, Somalia und Nigeria) stammen die meisten Geflüchteten in Deutschland. 


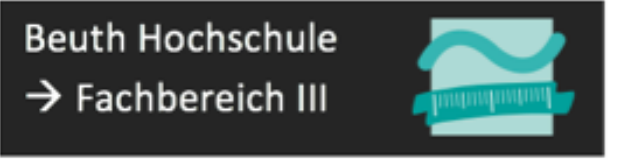

Wählen Sie die Übersetzungsrichtung aus:

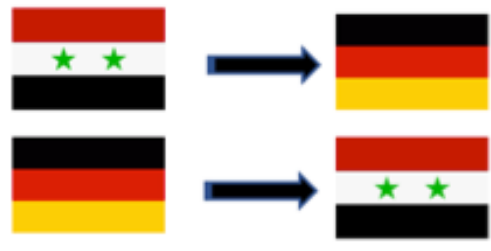

Geben Sie ihren Suchbegriff in das Feld ein:

Beton

\section{Direkt zur Gesamtübersicht}
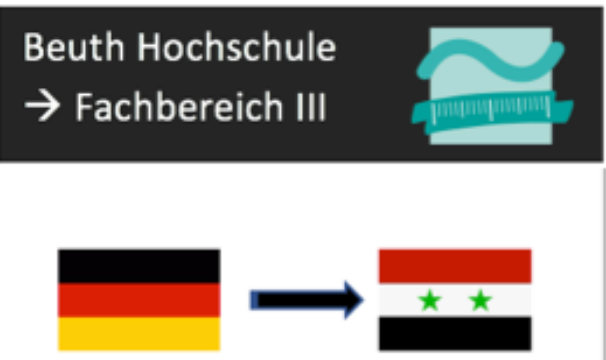

SUCHERGEBNIS:

\begin{tabular}{l|l} 
Deutsch & Arabisch \\
\hline Beton $(0))$ & asmant \\
&
\end{tabular}

Listen-Erweiterungsvorschlag abgeben
Bevor der fachspezifische Suchbegriff in das entsprechende Suchfeld eingegeben werden kann, muss die gewünschte Übersetzungsrichtung ausgewählt werden - je nachdem, ob der_die Studierende einen deutschen Fachbegriff nachschlagen möchte (z.B. um die Vorlesung nachzuvollziehen) oder ob er_sie einen Fachbegriff in der jeweiligen Muttersprache nachschlagen möchte (z.B. um sich bei Gruppenarbeiten einzubinden). Wir haben einen Button unter dem Suchfeld platziert, der es ermöglicht, auch direkt auf die Gesamtübersicht der Fremdwörterliste des ausgewählten Fachbereichs zuzugreifen.
Schließlich wird der gesuchte Fachbegriff in der gewünschten Sprache angezeigt. Des Weiteren kann die eigene Aussprache durch die Lautsprecherfunktion verbessert werden. Bezüglich der Frage: „Wer garantiert die Aktualität der Fremdwörterliste bzw. die ordnungsgemäße Übersetzungsleistung?" ist unser Vorschlag, dass studentische Hilfskräfte zum Einsatz kommen, die nicht nur die Liste mit Fachbegriffen füllen, sondern auch die Listen-Erweiterungsvorschläge zertifizieren (Übersetzungsvorschläge über $\mathrm{App}$ und Smartboard möglich). 
Die Idee einer fachspezifischen Datenbank mit fachbezogenen Begrifflichkeiten scheint eine vielversprechende Grundidee $\mathrm{zu}$ sein. Positiv hervorzuheben ist, dass theoretisch durch eine fachbereichsübergreifende Zusammenarbeit der Kostenaufwand gering bleiben kann und die notwendigen entwicklungstechnischen Ressourcen, wie ehrenamtliche Studenten, Excel, Server zum Speichern der Daten etc., vorhanden sind.

Der zeitintensivste Schritt wird hierbei das "Füttern" der Datenbank sein, das durch das Anbieten spezieller Studium Generale-Kurse oder das Einstellen studentischer Hilfskräfte zu beschleunigen wäre. Ist erstmal eine Datenbank entstanden, sinkt der Verwaltungsaufwand deutlich $a b$, da sich Fachbegriffe im Allgemeinen nur langsam verändern.

Insgesamt ist davon auszugehen, dass die Datenbank den Einstieg in das Studium für geflüchtete Menschen erheblich erleichtern und einen erfolgreichen Studienabschluss fördern kann.

\section{Reflexion und Implikationen}

Mit diesem Beitrag werden die Erfahrungen aus der Lehrveranstaltung "Innovationsprojekt: Digitalisierung und Diversity Management" dargestellt. Dabei soll gezeigt werden: (1) Wie die Studierenden ihre Kompetenzen in den Themen Design Thinking, Digitalisierung und Diversity Management weiterentwickeln konnten und inwiefern das Lehrkonzept und die Prüfungsformen dabei förderlich waren, und (2) welche Lösungen die Studierenden zu dem Thema digitale Unterstützung von Vielfalt an der Beuth Hochschule für Technik Berlin innerhalb eines Semesters erarbeitet haben. Entsprechend gliedert sich dieses Kapitel in zwei Abschnitte zu den Aspekten Kompetenzentwicklung der Studierenden und entwickelte Lösungen.

\section{Kompetenzentwicklung der Studierenden}

Die Lehrveranstaltung "Innovationsprojekt" im Studium Generale hat zum Ziel, die fachlichen, methodischen, persönlichen und sozialen Kompetenzen der Studierenden zu fördern und ihnen grundlegende Kenntnisse in den Themen Design Thinking, Digitalisierung und Diversity Management zu vermitteln.

Da sich Kompetenz in nicht-beobachtbare (Disposition, Potenzial) und beobachtbare (Verhalten, Performanz) Elemente untergliedert (Schuler \& Barthelme 1995), ist es wichtig, die einzelnen Kompetenzen nicht nur beobachtbar, sondern auch bewertbar zu machen und dafür entsprechende Kriterien zu entwickeln. Deshalb wurden zur Gewinnung von beobachtbaren Verhalten der Studierenden zwei Prüfungsformen genutzt, welche als Indikatoren Rückschlüsse auf deren Kompetenz erlauben. Für beide Prüfungsformen wurden Standards in Bezug auf diese Indikatoren definiert, welche eine einheitliche Bewertung der Kompetenzen der Studierenden ermöglichen. Des Weiteren wurde die Lehrveranstaltung am Ende des Semesters durch die Studierenden evaluiert, welches eine weitere Basis für die Einschätzung der Kompetenzentwicklung bildet.

Im Folgenden werden zunächst die beiden Prüfungsformen vorgestellt, um darauf aufbauend die Bewertungskriterien für die Kompetenz der Studierenden zu erläutern, um sich dann auf die studentischen Essays und inwiefern die Studierenden ihre Arbeit im "Innovationsprojekt" reflektieren können zu fokussieren.

- Prüfungsformen: Aus Sicht der Dozent_innen hat es sich als zielführend erwiesen, dass die Prüfungsleistung für die Lehrveranstaltung "Innovationsprojekt" in zwei Prüfungsformen unterteilt war: zum einen konnten die Studierenden dadurch nicht nur unterschiedliche 
Kompetenzen trainieren, z.B. durch die Abschlusspräsentation als Prüfungsform wird die Präsentationsfähigkeiten der Studierenden trainiert, wohingegen das Erstellen eines Essays die Schreibkompetenzen der Studierenden trainiert (beides Methodenkompetenzen). Zum anderen konnte mittels der zwei Prüfungsformen die Kompetenzentwicklung unterschiedlich überprüft werden, denn in den Essays ist ein bewertbares Kernelement, inwiefern die Studierenden in der Lage sind ihre Essays reflektiert zu verfassen, d.h. über die Inhalte und Ergebnisse reflektieren können (Persönliche Kompetenz). Beide Prüfungsleistungen waren als Teamleistung konzipiert, wodurch zudem die Teamkompetenz trainiert werden sollte (Sozialkompetenz). Dabei war es eine Anforderung, dass beide Prüfungsformen fachspezifisches Wissen zu den Themen Design Thinking, Digitalisierung und Diversity Management enthalten (Fachkompetenz). In diesem Beitrag wird sich zunächst auf die studentischen Essays konzentriert und dabei insbesondere auf die Reflexionsfähigkeit als Persönliche Kompetenz, da dies ein gut beobachtbares und bewertbares Kriterium ist. Anschließend wird auf die weiteren Kompetenzen auf Basis der Evaluationsergebnisse der Lehrveranstaltung durch die Studierenden eingegangen.

- Vorstellung der Bewertungskriterien für studentische Essays: Um die studentischen Essays über das Innovationsprojekt zu analysieren, wurden die Reflexionsniveaus von Neville Hatton und David Smith (1995) genutzt, wonach sich die schriftlichen Gruppenarbeiten der Studierenden in folgende Reflexionsebenen in aufsteigendem Anspruchsniveau unterscheiden können: (1) descriptive writing, (2) descriptive reflection, (3) dialogic reflection und (4) critical reflection.

Zum Erkennen der verschiedenen Arten von reflektiertem Schreiben werden die folgenden Kriterien definiert: (1) Auf dem Niveau von descriptive writing findet keine Reflexion statt, die Ereignisse werden lediglich beschrieben und es werden keine Gründe und Rechtfertigungen für die Ereignisse geliefert. (2) Auf dem Reflexionsniveau descriptive reflection zeichnet sich der Text durch eine (erste) Identifikation der Ursachen für die Ereignisse aus, allerdings in einen beschreibenden Stil. Diese Reflexion kann auf persönlichen Urteilen oder Literaturrecherche beruhen. (3) Wenn dialogic reflection vorliegt, dann hat eine Art Diskurs mit sich selbst über das Ereignis stattgefunden und dabei wurden mögliche Ursachen dargelegt. Es werden auch das Für und Wider der Erkenntnisse abgewogen und daraus alternative Erklärungsansätze und Annahmen abgeleitet worden. (4) Auf der höchsten Reflexionsstufe, dem critical reflection, werden die Entscheidungen begründet, auch unter Einbezug politischer, historischer und/oder sozialer Überlegungen und somit findet eine Verortung der Ereignisse im jeweiligen Kontext statt. Dabei könnten auch die Ziele selbst reflektiert werden (Hatton \& Smith 1995, S. 40 ff.). Dieses Model von Hatton und Smith (1995) zu den Reflexionsniveaus dient als Referenzrahmen für die Bewertung der vier studentischen Essays, welche als Indikatoren für eine beobachtbare Kompetenz der Studierenden genutzt wurden.

- Bewertung der studentischen Essays über das "Innovationsprojekt": In Kapitel 4 wurden die jeweiligen Ergebnisteile über die entwickelten Lösungen aus den vier studentischen Essays dargestellt. Dabei fällt auf, dass sowohl dieser Teil, wie auch die restlichen 13 bis 14 Seiten Text der Studierenden, vornehmlich dem descriptive writing sowie der descriptive reflection zuzuordnen sind. Sowohl Inhalte, welche sich auf das Thema Design Thinking beziehen, als auch Inhalte zu den Themen Digitalisierung und Diversity Management wurden vorwiegend beschrieben und nur teilweise reflektiert.

Die Chance das Erlebte zu verschriftlichen und sich dabei im Team auszutauschen hat sich aus Sicht der Autor_innen trotzdem bewährt. Für ein intensiveres Lernerlebnis ist es jedoch wichtig, bei einer nochmaligen Durchführung der Lehrveranstaltung, den Studierenden eine ausführlichere Erläuterung der verschiedenen Reflexionsniveaus inklusive Beispielen zur Verfügung zu stellen sowie diese Art des Denkens und Schreibens mit ihnen zu üben. 
In Bezug auf die drei Themenbereiche Design Thinking, Digitalisierung und Diversity Management ergeben sich des Weiteren folgende Kompetenzentwicklungen für die Studierenden, welche durch ihre Teilnahme an der Lehrveranstaltung "Innovationsprojekt" ermöglicht wurden. Inwieweit die Studierenden ihre verschiedenen Kompetenzen in diesen Bereichen weiterentwickelt haben, kann auf zweierlei Art beantwortet werden: zum einen auf Basis der Prüfungsleistungen, und damit aus der Perspektive der Autoren_innen, und zum anderen aus Sicht der Studierenden selbst mittels der Evaluation der Lehrveranstaltung. Die Evaluation der Lehrveranstaltung wird an der Beuth Hochschule für Technik Berlin durch die Studierenden vorgenommen, welche dafür einen Fragebogen vom Qualitätsmanagement der Hochschule bekommen. Dieser Fragebogen umfasst 20 items, welche auf einer Skala von 1 (sehr gut) bis 5 (mangelhaft) bewertet werden können sowie die Möglichkeit Freitext-Antworten zu geben. Die Studierenden haben die Lehrveranstaltung zusammenfassend mit einer 1,1 (sehr gut) bewertet:

- Kompetenzen zu Design Thinking: Die Studierenden konnten durch die Teilnahme an der Lehrveranstaltung wichtige Methodenkompetenzen zur Problemlösung und Innovationsentwicklung erwerben und ihre Fachkompetenzen zum Thema Design Thinking ausbauen. Sowohl die Abschlusspräsentationen als auch die studentischen Essays waren Indikatoren dafür, dass die Studierenden ein initiales Verständnis für Design Thinking erworben haben, z.B. in Form ihrer schriftlichen Darstellung der einzelnen Prozessschritte (Fachkompetenz) oder ihre Erkenntnisse aus der Durchführung von qualitativer und nutzer_innenzentrierter Datenerhebung (Methodenkompetenz). In den Evaluationsbögen wurden dazu vermerkt "ich weiß jetzt was Design Thinking ist" (Fachkompetenz) sowie "ich habe die Methode endlich verstanden" (Methodenkompetenz). Des Weiteren wurde die Persönliche Kompetenz gestärkt, indem die Studierenden vermerkt haben "mein Interesse für ein weiteres Selbststudium im Bereich Innovation wurde geweckt". Da sich die Arbeit mit der Methode Design Thinking auch durch eine diverse, insbesondere multidisziplinäre und hierarchiefreie Teamarbeit auszeichnet, wurde dadurch die Fähigkeit zur Gestaltung sozialer Interaktion, der einzelnen Studierenden gefördert (Sozialkompetenz). Dazu wurden insbesondere die "sehr guten Teambuilding-Maßnahmen" im Evaluationsbogen hervorgehoben.

- Kompetenzen zu Digitalisierung: Die Studierenden in der Lehrveranstaltung konnten sich durch ihre Teilnahme am "Innovationsprojekt" digitale Kompetenzen aufbauen, indem sie als Team an digitalen Lösungen gearbeitet haben. Dabei wird durch die unterschiedlichen von den Studierenden entwickelten digitalen Lösungen von App, Website, Datenbank bis zu einem digitalen Terminal deutlich, dass die Studierenden dafür sensibilisiert wurden (Fachund Methodenkompetenzen), dass die Wahl der geeigneten digitalen Anwendung wichtig für die bestmögliche Bearbeitung der Aufgabe ist. Somit konnte die digitale Fitness als digitale Schlüsselkompetenz der einzelnen Studierenden gefördert werden. Zudem zeichnet sich auch die Digitalisierung durch eine meist radikale Nutzer_innen-bzw. Kunden_innenzentrierung aus, weshalb die Studierenden mittels der Design Thinking Methode ein Art der Umsetzung kennengelernt haben und gleichzeitig erfahren haben, wie der_die Nutzer_in - in diesem Fall die Geflüchteten - determinieren, welche (digitale) Lösung wie ausgestaltet werden soll und wie zum Beispiel die unterschiedlichen Touchpoints von digitalen Lösungen aussehen können.

- Kompetenzen zu Diversity Management: Während der Lehrveranstaltung "Innovationsprojekt" haben die Studierenden in diversen, multidisziplinären Teams zusammengearbeitet und konnten in den unterschiedlichen Phasen vom Problemverständnis bis zur Lösungsfindung erleben, welche Vorteile unterschiedliche Perspektiven aufgrund von Vielfalt in der Teamzusammensetzung haben können (Persönliche Kompetenz). Gleichzeitig wurden durch diese Teamarbeit die Sozialkompetenzen der einzelnen Teammitglieder gestärkt, 
d.h. Fähigkeiten zu sozialen Interaktionen in vielfältigen Teams. Zweitens haben die Studierenden ein Semester intensiv an Lösungen zur Unterstützung von Vielfalt an der Hochschule gearbeitet und sich dabei (teilweise) zum ersten Mal in ihrem Studium mit Diversity Management an der Hochschule auseinandergesetzt (Fachkompetenz). Dadurch werden die Studierenden idealerweise auch zur Selbstreflexion über ihre eigene Diversitätsidentität angeregt (Persönliche Kompetenz). Gleichzeitig bestand durch die nutzer_innenzentrierte Arbeitsweise die Möglichkeit Empathie und Verständnis für die Geflüchteten an der Hochschule zu entwickeln. Die Studierenden haben qualitative Interviews mit verschiedenen geflüchtete Menschen geführt sowie ihre erarbeiteten Ergebnisse gemeinsam mit ihnen getestet. Dies kann nicht nur dazu führen die eigenen diversitätsbezogenen Denk- und Handlungsmuster zu hinterfragen und dadurch die persönliche Kompetenz auszubauen, sondern auch die Relevanz von Unterstützungsangeboten für Geflüchtete als Teil des Diversity Managements zu erkennen.

\section{Entwickelte Lösungen}

Die innovativen Lösungen der Studierenden zur digitalen Unterstützung von Vielfalt an der Beuth Hochschule für Technik Berlin verdeutlichen, wie mittels der Design Thinking Methode digitale Technologielösungen entwickelt werden können, um Geflüchtete bei ihrem Studienalltag zu unterstützen und damit Diversity Management an der Hochschule nutzer_innenzentriert umgesetzt werden kann.

- Design Thinking-Ebene der Lösung: Design Thinking ermöglicht es, Diversity Management an der Hochschule nutzer_innenzentriert auszugestalten. Die vier vorgestellten digitalen Lösungen adressieren jeweils andere Bedürfnisse oder Probleme der Nutzer_innengruppe "Geflüchtete". Dabei zeigt sich, dass sich bereits innerhalb der Gruppe Geflüchteter die Bedürfnisse unterscheiden und die (digitalen) Unterstützungsangebote von den Studierenden entsprechend unterschiedlich ausgestaltet wurden. Deshalb ist es für das Diversity Management an der Hochschule wichtig, jeweils auf die Bedürfnisse der einzelnen Diversitätsgruppen einzugehen und $\mathrm{zu}$ analysieren, wie die Unterstützungsangebote ausgestaltet werden müssen um die Vielfalt an der Hochschule gezielt zu fördern. Zum Beispiel ist davon auszugehen, dass Frauen an der Hochschule andere Unterstützungsangebote benötigen, als ältere Studierende oder Studierende mit körperlichen oder geistigen Einschränkungen. Design Thinking ist dabei eine Methode, um diese Unterstützungsangebote auf die jeweilige Nutzer_innengruppe zuzuschneiden.

- Digitalisierungsebene der Lösung: Den unterschiedlichen Lösungen liegen verschiedene digitale Ausgestaltungen zugrunde. Es existiert eine Vielzahl an Möglichkeiten, Produkte oder Services digital umzusetzen. Je nach Bedürfnis der Nutzer_innen variieren die von den Studierenden entwickelten Lösungen dabei von App über Website, Datenbank bis zu einem digitalen Terminal. Es wird deutlich, dass die Studierenden dafür sensibilisiert wurden, dass die Wahl der geeigneten Anwendung wichtig für die bestmögliche Umsetzung der Lösung und Adressierung des Bedürfnisses der Nutzer_innen ist.

- Diversitätsebene der Lösung: Der Umgang mit Diversität wird an der Hochschule unmittelbar verbessert, indem die geflüchteten Studierenden durch die entwickelten digitalen Lösungen in ihrem Studienalltag unterstützt werden und sich dadurch deren Erfolg an der Hochschule erhöhen kann. Indem die Geflüchteten und deren Bedürfnisse die Grundlage für die vier vorgestellten Lösungen - Website „Refugees Reiter“, App „BeutHelp“, Digitaler Terminal „BE(uth) Connected“ und Datenbank "Termynl” - waren, ist es wahrscheinlich, dass die Umsetzung dieser Lösungen eine Unterstützung in deren Studienalltag bedeuten kann.

Insgesamt lässt sich festhalten, dass die von den Studierenden entwickelten Lösungen, welche in den studentischen Essays beschrieben wurden, interessante Ansätze zur digitalen Unterstützung von 
Vielfalt an der Beuth Hochschule für Technik Berlin liefern. Gleichzeitig kann die Kombination der drei Themen Design Thinking, Digitalisierung und Diversity Management in der beschriebenen Lehrveranstaltung zu einer zukunftsgerichteten fachlichen, methodischen, persönlichen und sozialen Kompetenzbildung der Studierenden beitragen. Für derartige Lehrveranstaltungen ist es gewinnbringend sie im fachbereichsübergreifenden Studium Generale anzusiedeln, um eine diverse Studierendenschaft anzusprechen und dadurch ein Lernerlebnis in einem multidisziplinären Team zu ermöglichen - welches ein zentrales Prinzip von Design Thinking ist und auch für die Sensibilisierung im Bereich Diversity hilfreich sein kann. Dabei ist die Gestaltung als Übung und nicht Vorlesung entscheidend, um die Lernenden in den Mittelpunkt zu stellen und ihnen reale Probleme zum Lösen zu geben (Tschepe 2018).

Das dargelegte Fallbeispiel zeigt, wie Hochschullehre Studierende auf die neuen Anforderungen der digitalen Arbeitswelt nicht nur inhaltlich, sondern auch methodisch konkret und erlebnisreich vorbereiten kann. 


\section{Literatur}

Afflerbach, T. \& Gläsener, K. (2016). New Ways of Working - Vertrauen und Selbstmanagement in einer digitalisierten Arbeitswelt. In: Badura B., Ducki A., Schröder H., Klose J. \& Meyer M. (Hrsg.). Fehlzeiten-Report 2016. Vielfalt managen: Gesundheit fördern - Potenziale nutzen, Springer Verlag, Berlin, Heidelberg.

Aretz, H.J. \& Hansen, K. (2003). Erfolgreiches Management von Diversity. Die multikulturelle Organisation als Strategie zur Verbesserung einer nachhaltigen Wettbewerbsfähigkeit. Zeitschrift für Personalforschung 17(1), S. 9-36.

Auferkorte-Michaelis, N. \& Linde, F. (2016). Diversity Management an Hochschulen. In: Genkova, P. \& Ringeisen, T. (Hrsg.). Handbuch Diversity Kompetenz - Perspektiven und Anwendungsfelder. Springer Verlag, Berlin, Heidelberg.

Bender, S.-F., Schmidbaur, M. \& Wolde, A. (2013). Zur Einleitung: Diversity Policies - Ein Beitrag zur Öffnung der Hochschulen?! In: Bender, S.-F., Schmidbaur, M. \& Wolde, A. (Hrsg.). Diversity entdecken - Reichweiten und Grenzen von Diversity Policies an Hochschulen. Beltz Juventa, Weinheim und Basel, S. 7-12.

Beuth Hochschule (2018a). Allgemeinwissenschaftliches Wahlpflichtangebot, http://www.beuthhochschule.de/192/ (abgerufen am 01.04.2018).

Bitkom (2015). Arbeit 3.0 - Arbeiten in der digitalen Welt. Berlin: Bundesverband Informationswirtschaft, Telekommunikation und neue Medien e.V.

Blatt M. \& Sauvonnet E. (2017). Wo ist das Problem? Mit Design Thinking

Innovationen entwickeln und umsetzen, 2. komplett überarbeitete Auflage. Verlag Franz Vahlen, München.

BPB (2016). Die digitale Welt von heute und morgen. Bundeszentrale für politische Bildung. http://www.bpb.de/apuz/225685/die-digitale-arbeitswelt-von-heute-und-morgen (abgerufen am 10. 05.2018).

Brenner, W., Uebernickel, F. \& Abrell, T. (2016). Design Thinking as Mindset, Process, and Toolbox. In: Brenner, W. \& Uebernickel, F. (Hrsg.). Design Thinking for Innovation. Springer International Publishing, Switzerland, S. 3-21.

Brown, T. (2009). Change by Design - How Design Thinking Transforms Organizations and Inspires Innovation. New York, Harper Business.

DAAD (2018). Wissenschaft weltoffen - Daten und Fakten zur Internationalität von Studium und Forschung in Deutschland. Wbv Media, Bielefeld.

De Leede, J. \& Kraijenbrink, J. (2014). The Mediating Role of Trust and Social Cohesion in the Effects of New Ways of Working: A Dutch Case Study. Human Resource Management, Social Innovation and Technology, Advanced Series in Management 14, S. 3-20.

Derks, D. \& Bakker, A.B. (2010). The impact of e-mail communication on organizational life. Cyberpsychology. Journal of Psychosocial Research on Cyberspace 4(1), S. 1-16.

DGFP (2016). Leitfaden - Kompetenzen im digitalisierten Unternehmen. 02/2016, Deutsche Gesellschaft für Personalführung e.V., Frankfurt a. M. 
Ducki, A. (2016). Smart arbeiten - Arbeitspsychologische Gestaltungsoptionen für multiple Entgrenzungen. In. R. Wieland, K. Seiler \& M. Hammes (Hrsg.), Psychologie der Arbeitssicherheit und Gesundheit Dialog statt Monolog. Kröning: Asanger Verlag, S. 15-25.

Duden (o.J.). Design. https://www.duden.de/rechtschreibung/Design (abgerufen am 05.05.2018).

Dunne, D. \& Martin, R. (2006). Design Thinking and how it will change management education: An interview and discussion. Academy of Management Learning \& Education 5(4), S. 512-523.

Ely, R.J. \& Thomas, D.A. (2001). Cultural diversity at work: The effects of diversity perspectives on work group processes and outcomes. Administrative Science Quarterly 46(2), S. 229-273.

Erbeldinger, J. \& Ramge, T. (2015). Durch die Decke denken. Design Thinking in der Praxis. 3. Auflage. Redline Verlag, München.

Grots, A. \& Pratschke, M. (2009). Design Thinking - Kreativität als Methode. Marketing Review St. Gallen 26(2), S. 18-23.

Gürtler, J. \& Meyer, J. (2013). Design Thinking - 30 Minuten. Gabal Verlag, Offenbach.

Hatton, N., \& Smith, D. (1995). Reflection in teacher education- Towards definition and implementation. Teaching and teacher education 11(1), S. 33-49.

HPI (2018a). Glossar, https://hpi-academy.de/journal/glossar.html (abgerufen am 02. März 2018).

Hochschulrektorenkonferenz (2009). Eine Hochschule für Alle. Empfehlung der 6. Mitgliederversammlung am 21.4.2009. Zum Studium mit Behinderung/chronischer Krankheit. http://www.hrk.de/uploads/tx_szconvention/Entschliessung_HS_Alle.pdf (abgerufen am 02.03.2018).

Johns, T. \& Lynda Gratton, L. (2013). The third wave of virtual work." Harvard Business Review 91(1), S. 66-73.

Kauffeld, S., Grote, S. \& Frieling, E. (2011). Handbuch Kompetenzentwicklung. Schäffer-Poeschel Verlag, Stuttgart.

Klein, U. (2013). Diversityorientierung und Hochschulen im Wettbewerb. Ein Plädoyer für Diversitypolitik. In: Bender, S.-F., Schmidbaur, M. \& Wolde, A. (Hrsg.). Diversity entdecken Reichweiten und Grenzen von Diversity Policies an Hochschulen. Beltz Juventa, Weinheim und Basel, S. 79-96.

Krell, G., Riedmüller, B., Sieben, B. \& Vinz, D. (2007). Einleitung - Diversity Studies als integrierende Forschungsrichtung. In: Krell, G., Riedmüller, B., Sieben, B. \& Vinz, D. (Hrsg.) Diversity Studies - Grundlagen und disziplinäre Ansätze. Frankfurt und New York: Campus.

Lederle, S. (2007). Die Einführung von Diversity Management in deutschen Organisationen: Eine neoinstitutionalistische Perspektive. In: Zeitschrift für Personalforschung 21(1), 22-41.

Legner, C., Eymann, T., Hess, T., Matt, C., Böhmann, T., Drews, P., Mädche, A., Urbach, N. \& Ahlemann, F. (2017). Digitalization: Opportunity and Challenge for the Business and Information Systems Engineering Community, Business and Information Systems Engineering 59(4), S. 301-308.

Lindberg, T., Meinel, C., \& Wagner, R (2011). Design Thinking - A Fruitful Concept for IT Development? In: Plattner, H., Meinel, C., \& Leifer, L. (Hrsg.). Design thinking - Understand, Improve, Apply. Springer Verlag, Berlin Heidelberg, S. 4-18.

Link, P. (2014). Agile Methoden im Produkt-Lifecycle-Prozess - Mit agilen Methoden die Komplexität im Innovationsprozess handhaben. In: Schoeneberg, K.-P. (Hrsg.). 
Komplexitätsmanagement in Unternehmen - Herausforderungen im Umgang mit Dynamik, Unsicherheit und Komplexität meistern. Springer Gabler Verlag, Wiesbaden.

Meifert, M. T. (2013). Strategische Personalentwicklung. Springer Verlag, Wiesbaden.

Meinel, C., Weinberg, U. \& Krohn, T. (2015). Design Thinking Live. Wie man Ideen entwickelt und Probleme löst. Murmann Publishers, Hamburg.

Schallmo, D. R. (2017). Design Thinking erfolgreich anwenden. So entwickeln Sie in 7 Phasen kundenorientierte Produkte und Dienstleistungen. Springer Gabler Verlag, Wiesbaden.

Schuler, H. \& Barthelme, D. (1995). Soziale Kompetenz als berufliche Anforderung. In: Seyfried, B. (Hrsg.). "Stolperstein" Sozialkompetenz - Was macht es so schwierig, sie zu erfassen, zu fördern und zu beurteilen? Berichte zur beruflichen Bildung, Bd. 179. Bertelsmann, Bielefeld, S. 77-116.

Stangl, W. (2001). Der Begriff der sozialen Kompetenz in der psychologischen Literatur (Version 2.0), http://paedpsych.jk.uni-linz.ac.at/PAEDPSYCH/SOZIALEKOMPETENZ/ (abgerufen am 14.04.2018)

Statista (2018). Anteil ausländischer Studierender an deutschen Hochschulen vom Wintersemester 1998/1999 bis 2016/2017,

https:/de.statista.com/statistik/daten/studie/222/umfrage/anteil-auslaendischer-studenten-anhochschulen/ (abgerufen am 12.05.2018)

Tilson, D., Lyytinen, K. \& Sørensen, C. (2010). Digital infrastructures: the missing IS research agenda. Information Systems Research 21(4), S. 748-759

Tschepe, S. (2018). How Design Thinking can benefit education. https://medium.com/swlh/howdesign-thinking-can-benefit-education-2bba35450771 (abgerufen am 20.08.2018)

Uebernickel, F., Brenner, W., Pukall, B., Naef, T. \& Schindlholzer, B. (2015). Design Thinking - Das Handbuch. Frankfurter Allgemeine Buch, Frankfurt am Main.

Weigert, M., Bruhn, H. D., \& Strenge, M. (2017). Digital HR oder HR Digital - Die Bedeutung der Digitalisierung für HR. In: Jochmann, W., Böckenholt, I. \& Diestel, S. (Hrsg.). HR-Exzellenz. Springer Gabler, Wiesbaden. S. 323-337.

Weinberg, U. (2015). Network Thinking - Was kommt nach dem Brockhaus-Denken? Murmann Publishers, Hamburg.

Wissenschaftsrat (Hrsg.). (2013). Perspektiven des deutschen Wissenschaftssystems. Drs. 3228-13. http://www.wissenschaftsrat.de/download/archiv/3228-13.pdf (abgerufen am 12. Mai 2018). 


\section{Bisher erschienene Bände der Schriftenreihe des GuTZ:}

Jahrgang 2009

Ausgabe 01/2009:

Jahrgang 2010

Ausgabe 02/2010:

Ausgabe 03/2010:

Jahrgang 2011

Ausgabe 04/2011:

Jahrgang 2012

Ausgabe 05/2012:

Jahrgang 2013

Ausgabe 06/2013:

Jahrgang 2014

Ausgabe 07/2014:
Barthel, K. (Dez. 2009). Wer wagt, gewinnt? Geschlechtsspezifische Unterschiede im Entscheidungsverhalten unter Risiko. http://www.beuthhochschule.de/fileadmin/oe/gutz/Schriftenreihe/GuTZ-Schriftenreihe_01_122009_Barthel.pdf

Pattloch, A. (Dez. 2010). "Studieren in der Lounge" - Wie StudentInnen Ihre Hochschule gestalten würden. Eine geschlechterdifferenzierende Exploration. http://www.beuth-hochschule.de/fileadmin/oe/gutz/Schriftenreihe/GuTZSchriftenreihe_02_12-2010_Pattloch.pdf

Ehmsen, S. (Dez. 2010). Die Vielfalt gestalten - Diversity an Hochschulen. http://www.beuth-hochschule.de/fileadmin/oe/gutz/Schriftenreihe/GuTZSchriftenreihe_03_02-2010_Ehmsen.pdf

Gläsener, K. (April 2011). Diverse Teams = Erfolgsteams? Bedingungen für die Interaktion in geschlechts- und nationalitätsgemischten Teams. http://www.beuth-hochschule.de/fileadmin/oe/gutz/Schriftenreihe/GuTZSchriftenreihe_04_04-2011_Glaesener.pdf

Ihsen, S. und Ducki, A. (Juli 2012). Gender Toolbox. http://www.beuthhochschule.de/fileadmin/oe/gutz/Schriftenreihe/GuTZ-Schriftenreihe_05_072012_Ihsen-Ducki_Gender_Toolbox.pdf

Erlemann, C. (Dez. 2013). Studentinnen mit Migrationshintergrund in Chancengleichheitsprojekten. http://www.beuthhochschule.de/fileadmin/oe/gutz/Schriftenreihe/GuTZ-Schriftenreihe_06_122013_Erlemann.pdf

Buchem, I. (Jan. 2014). Studentische Essays zum Thema Internet und Gesellschaft. http://www.beuthhochschule.de/fileadmin/oe/gutz/Schriftenreihe/GuTZ-Schriftenreihe_07_012014_Buchem.pdf

\section{Neu im Verlag Barbara Budrich:}

Jahrgang 2017

Ausgabe 08/2017:

Brandt, M. und Ducki, A. (2017). Gendersensible Gestaltung des neuen Studiengangs „BWL - Digitale Wirtschaft“ an der Beuth Hochschule für Technik Berlin. Verlag Barbara Budrci, Leverkusen.http://www.beuthhttps://shop.budrich-academic.de/produkt/gendersensible-gestaltung-desneuen-studiengangs-bwl-digitale-wirtschaft-an-der-beuth-hochschule-fuertechnik-berlin/?v=3a52f3c22ed6 
Ausgabe 09/2018: $\quad$ Diallo-Ruschhaupt, U., Plaumann, S. und Dombrowski, E.-M. (2018).

Karrierewege zu einer Professur an einer Fachhochschule. Verlag Barbara Budrich, Leverkusen. https://shop.budrich-

academic.de/produkt/karrierewege-zur-professur-an-einer-

fachhochschule/?v=3a52f3c22ed6 

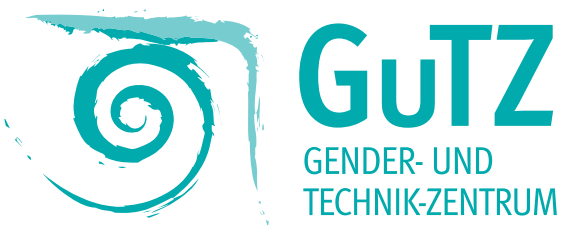

Die Gewinnung von FH-Professorinnen und Professoren wird seit 2016 von Wissenschaftsorganisationen intensiv diskutiert und bundesweit nach neuen Ideen und Ansätzen gesucht, das sogenannte Nachwuchsproblem zu lösen. Gelingt es an der Beuth Hochschule für Technik Berlin im Rahmen der freien Berufungskorridore den Frauenanteil unter den FH-Professuren markant zu erhöhen und eine „place to be“-Strategie zu etablieren? Aufbauend auf den Erkenntnissen von 22 Jahren Förderung des wissenschaftlichen Nachwuchses im Hypatia Programm wird unter Berücksichtigung von Handlungsempfehlungen zum Personal Recruiting im Wissenschaftsbereich die besondere Situation von Hochschulen der angewandten Wissenschaften im MINT-Bereich berücksichtigt und im Rahmen des Gleichstellungskonzeptes mit Maßnahmen zum Karriereziel FH-Professorin verankert.

Schriftenreihe des Gender- und Technik-Zentrumsder Beuth Hochschule für Technik Berlin

\section{Herausgeberinnen:}

Prof. Dr. Eva-Maria Dombrowski eva-maria.dombrowski@beuth-hochschule.de

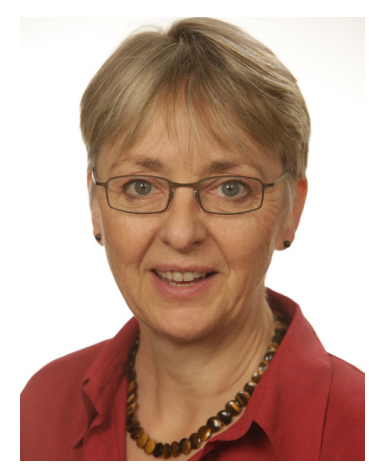

Prof. Dr. Antje Ducki ducki@beuth-hochschule.de
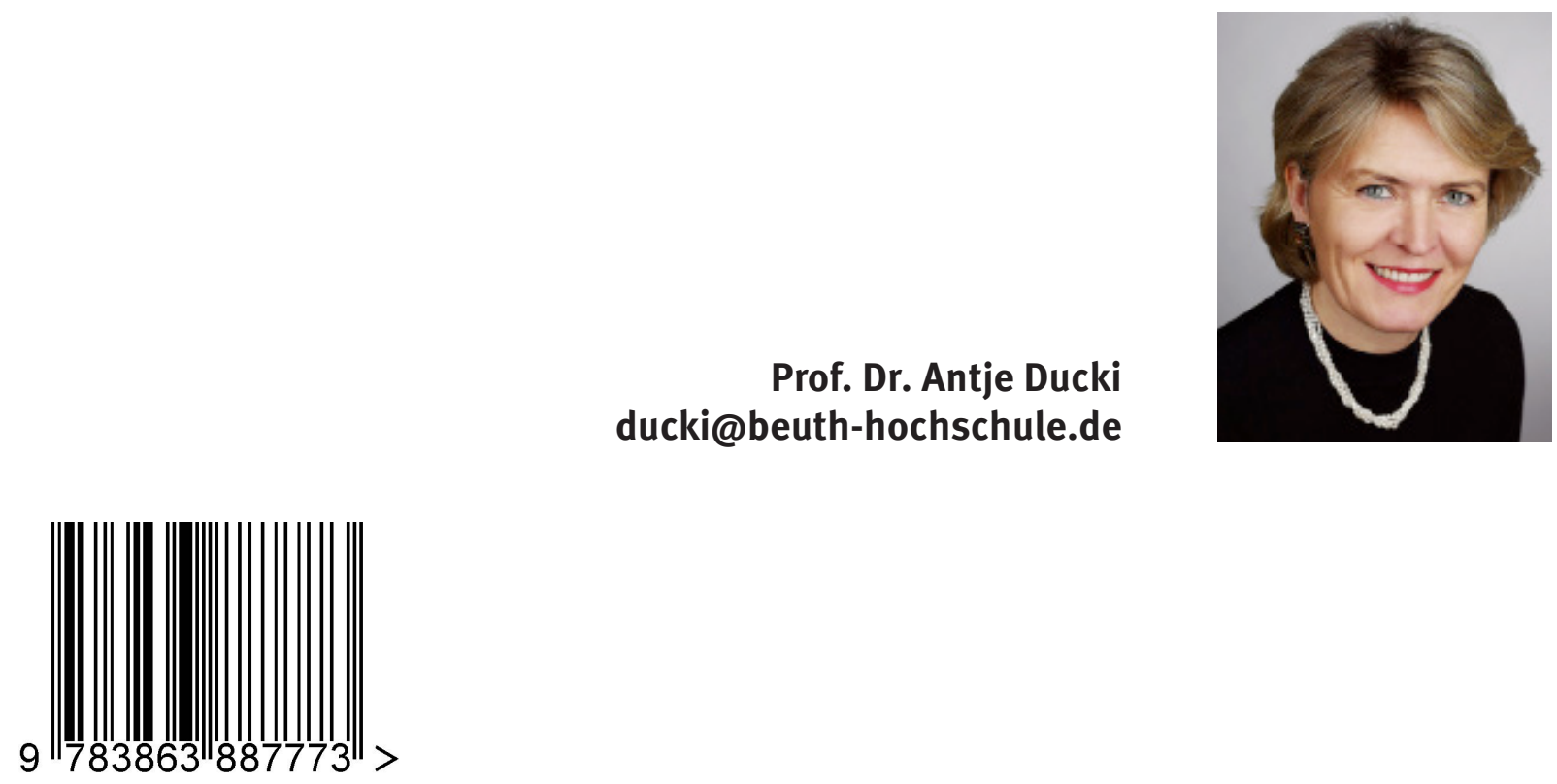